\title{
Conformal structure of minimal surfaces with finite topology
}

\author{
Jacob Bernstein* and Christine Breiner
}

\begin{abstract}
In this paper we show that a complete, embedded minimal surface in $\mathbb{R}^{3}$, with finite topology and one end, is conformal to a once-punctured compact Riemann surface. Moreover, using this conformal structure and the embeddedness of the surface, we examine the Weierstrass data and conclude that every such surface has Weierstrass data asymptotic to that of the helicoid. More precisely, if $g$ is the stereographic projection of the Gauss map, then in a neighborhood of the puncture, $g(p)=\exp (i \alpha z(p)+F(p))$, where $\alpha \in \mathbb{R}, z=x_{3}+i x_{3}^{*}$ is a holomorphic coordinate defined in this neighborhood and $F(p)$ is holomorphic in the neighborhood and extends over the puncture with a zero there. As a consequence, the end is asymptotic to a helicoid. This completes the understanding of the conformal and geometric structure of the ends of complete, embedded minimal surfaces in $\mathbb{R}^{3}$ with finite topology.
\end{abstract}

Mathematics Subject Classification (2010). 53A10; 49Q05.

Keywords. Complete embedded minimal surface, helicoid, conformal type.

\section{Introduction}

We apply the techniques of [1] to study complete, embedded minimal surfaces in $\mathbb{R}^{3}$ with finite topology and one end. We refer to the space of such surfaces as $\mathcal{E}(1)$. Notice that we do not a priori assume the surfaces are properly embedded. This is because Colding and Minicozzi have shown, in Corollary 0.13 of [8], that every complete, embedded minimal surface with finite topology is, in fact, properly embedded. This fact will be used implicitly throughout. Surfaces in $\&$ (1) that have genus zero have been completely classified by Meeks and Rosenberg in [23] and consist of planes and helicoids; thus we restrict attention to the subset $\mathscr{E}(1,+) \subset \mathcal{E}(1)$ of surfaces that have positive genus. This space is non-trivial; the embedded genus one helicoid, $\mathscr{H}$, constructed in [16] by Hoffman, Weber, and Wolf provides an example which, moreover, has the property of being asymptotically helicoidal (see also [26] for a nice overview of their construction).

The construction and study of $\mathscr{H}$ has a rich history. Using the Weierstrass representation, Hoffman, Karcher, and Wei in [14] first constructed an immersed genus-one

\footnotetext{
${ }^{*}$ The first author was supported in part by the NSF grant DMS-0606629.
} 
helicoid. See Figure 1 for an image of this surface. Computer graphics suggested it was embedded, but a rigorous construction of an embedded genus-one helicoid followed only after Hoffman and Wei proposed a new construction in [18]. They considered the limit of a family of screw-motion invariant minimal surfaces with periodic handles and a helicoidal end. Weber, Hoffman, and Wolf confirmed the existence of such a family of surfaces in [17] and ultimately proved their embeddedness in [16], giving $\mathscr{H}$. Hoffman, Weber, and Wolf conjecture that $\mathscr{H}$ is not only the same surface as the one produced in [14], but is actually the unique element in the class of "symmetric" genus-one helicoids, that is, surfaces in $\mathcal{E}(1)$ with genus one and containing two coordinate axes. Recently, Hoffman and White, in [19], used variational methods to give a different construction of a symmetric, embedded genus-one helicoid; whether their construction is $\mathscr{H}$ is unknown.

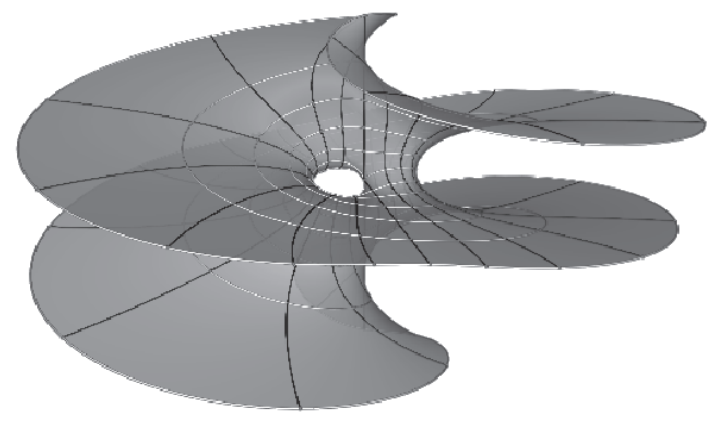

Figure 1. A genus-one helicoid (courtesy of Matthias Weber).

Building on [19], in [20], Hoffman and White prove rigidity results for properly immersed minimal surfaces with genus one and one end that, moreover, admit the same symmetries as $\mathscr{H}$. In particular, they show such surfaces are conformal to a punctured torus and are asymptotic to a helicoid. In this paper, we prove that any $\Sigma \in \mathcal{E}(1,+)$ is conformal to a once-punctured, compact Riemann surface and its Weierstrass data - see (1.1) - has helicoid-like behavior at the puncture:

Theorem 1.1. Any $\Sigma \in \mathcal{E}(1)$ is conformally a punctured, compact Riemann surface. Moreover, if the surface is not flat, then, after a rotation of $\mathbb{R}^{3}$, the height differential, $d h$, extends meromorphically over the puncture with a double pole, as does the meromorphic one form $\frac{d g}{g}$.

The proof of this result draws heavily on the fundamental work of Colding and Minicozzi on the geometric structure of embedded minimal surfaces in $\mathbb{R}^{3}$ [4], [5], [6], 
[7], [9]. Assuming only mild conditions on the boundaries, they give a description of the geometric structure of essentially all embedded minimal surfaces with finite genus. From this structure, they deduce a number of important consequences. These include: the one-sided curvature estimate for embedded minimal disks [7] - an effective version of the strong half-space theorem of Hoffman and Meeks [13]; a compactness result for a sequence of embedded minimal disks in [7] that requires no a priori bounds on the curvature or area; and the settling of the Calabi-Yau conjecture for embedded minimal surfaces of finite topology [8], i.e., a complete, embedded minimal surface in $\mathbb{R}^{3}$ of finite topology is properly embedded.

Colding and Minicozzi's work is also an essential ingredient in understanding minimal surfaces with infinite total curvature, i.e., complete surfaces with one end. Prior to their work, the study of these surfaces required very strong assumptions on the conformal structure and behavior of the Gauss map at the end. For examples we refer to Hoffman and McCuan [15] and Hauswirth, Perez and Romon [12]. The latter authors consider $E \subset \mathbb{R}^{3}$, a complete embedded minimal annulus with one compact boundary component and one end with infinite total curvature. They assume, in addition, that $E$ is conformal to a punctured disk, the Weierstrass data $(g, d h)$ has the property that $d g / g$ and $d h$ extend across the puncture, and the flux over the boundary of $E$ has zero vertical component. Assuming all of this, they then deduce more precise information about the asymptotic geometry of $E$. Notice in a suitable neighborhood of the puncture of a non-flat element of $\mathscr{E}(1)$, Theorem 1.1 immediately implies that these conditions are satisfied.

By using Colding and Minicozzi's work, in particular the compactness result of [7], Meeks and Rosenberg were able to remove such strong assumptions for disks. Indeed, in [23], they show that the helicoid is the unique non-flat complete, embedded minimal disk. They go on to discuss how the techniques of their proof might allow one to show something similar to Theorem 1.1 for surfaces in $\&(1,+)$ and the implications this has for the possible conformal structure of complete embedded minimal surfaces in $\mathbb{R}^{3}$. They do this without going into the details or addressing the difficulties, but indicate how such a statement may be proved using the ideas and techniques of their proof. In [1], we more directly use the geometric structure given by Colding and Minicozzi for embedded minimal disks to prove the uniqueness of the helicoid. In this paper, we generalize our argument to surfaces in $\mathcal{E}(1,+)$, thus determining the asymptotic structure of all elements of $\mathscr{E}(1)$.

Recall the Weierstrass representation takes a triple $(M, g, d h)$ where $M$ is a Riemann surface, $g$ is a meromorphic function and $d h$ is a meromorphic one form (which has a zero everywhere $g$ has a pole or zero) that satisfy certain natural compatibility conditions, and gives a minimal immersion of $M$ into $\mathbb{R}^{3}$ :

$$
\mathbf{F}:=\operatorname{Re} \int\left(\frac{1}{2}\left(g^{-1}-g\right), \frac{i}{2}\left(g^{-1}+g\right), 1\right) d h .
$$


Moreover, the immersion $\mathbf{F}$ is such that $\operatorname{Re} d h=\mathbf{F}^{*} d x_{3}$ and $g$ is the stereographic projection of the Gauss map of the image of $\mathbf{F}$. Any immersed minimal surface in $\mathbb{R}^{3}$ admits such a representation. For the helicoid with $z \in \mathbb{C}$ one has:

$$
g:=e^{i \alpha z} ; \quad d h:=d z ; \quad \alpha \in \mathbb{R}^{+} .
$$

Notice that on the helicoid both $\frac{d g}{g}$ and $d h$ have double poles at infinity; moreover, $\frac{d g}{g}-i \alpha d h$ is identically zero. For $\Sigma \in \mathcal{E}(1)$, Theorem 1.1, the Weierstrass representation, and embeddedness immediately imply that near the puncture the Weierstrass data is asymptotic to that of a helicoid. This is an immediate consequence of Theorem 1.1 above and Theorem 2 of [12], though we present our own proof in Section 4.3 (where we also prove Theorem 1.1). Indeed, we have:

Corollary 1.2. For $\Sigma$ as in Theorem 1.1, there exists an $\alpha \in \mathbb{R}$ so that $\frac{d g}{g}-i \alpha d h$ holomorphically extends over the puncture. Equivalently, after possibly translating parallel to the $x_{3}$-axis, in an appropriately chosen neighborhood of the puncture, $\Gamma \subset \Sigma, g(p)=\exp (i \alpha z(p)+F(p))$ where $F: \Gamma \rightarrow \mathbb{C}$ extends holomorphically over the puncture with a zero there and $z=x_{3}+i x_{3}^{*}$ is a holomorphic coordinate on $\Gamma$. Here $x_{3}^{*}$ is the harmonic conjugate of $x_{3}$.

Theorem 1 of [12] implies that for Weierstrass data $(\Gamma, g, d h)$ as in Corollary 1.2, that also satisfy a certain flux condition, the Weierstrass representation gives a minimal surface that is $C^{0}$-asymptotic to a (vertical) helicoid $H$. That is, for any $\epsilon>0$, there exists $R_{\epsilon}>0$, so that $\Gamma \backslash B_{R_{\epsilon}}(0)$ has Hausdorff distance to $H \backslash B_{R_{\epsilon}}(0)$ less than $\epsilon$. For elements of $\mathcal{E}(1)$, as the Weierstrass data is defined on a surface with only one end, this flux condition is automatically satisfied by Stokes' theorem. Thus, Theorem 1.1 allows one to immediately apply Theorem 1 of [12] and obtain:

Corollary 1.3. If $\Sigma \in \mathcal{E}(1)$ is non-flat then $\Sigma$ is $C^{0}$-asymptotic to some helicoid.

Theorem 1.1 and its corollaries complete the classification of the conformal type and asymptotic geometry of complete embedded minimal surfaces in $\mathbb{R}^{3}$ with finite topology. Indeed, let $\mathscr{E}(k)$ be the space of complete, embedded minimal surfaces with finite topology and $k$ ends. Meeks and Rosenberg, in Corollary 1.1 of [22], completely classify the conformal type of these surfaces when $k \geq 2$. Indeed, they show such a surface is conformal to a compact Riemann surface with $k$ punctures. However, they can only describe the asymptotic geometry at $k-2$ of the ends. Collin, in Theorem 1 of [10], overcomes this obstacle by proving that all such surfaces have finite total curvature. This, together with classic results of Huber [21] and Osserman [24], recovers not only the conformal type of surfaces in $\&(k)$ for $k \geq 2$, but also gives a description of their asymptotic geometry. Thus, he completes the classification for embedded minimal surfaces of finite topology and two or more ends. Combined with 
Theorem 1.1, we then have the following classification result for any minimal surface of finite topology that is complete and embedded in $\mathbb{R}^{3}$ :

Corollary 1.4. Let $\Sigma \in \mathcal{E}(k), k \geq 1$. Then $\Sigma$ is conformal to a punctured compact Riemann surface. Moreover, if $k \geq 2$, then $\Sigma$ has finite total curvature and each end of $\Sigma$ is asymptotic to either a plane or a catenoid. If $k=1$, then either $\Sigma$ is a plane or it has infinite total curvature and its end is asymptotic to a helicoid.

Let us now recall the argument of [1], where we provide an alternative proof to the uniqueness of the helicoid. There it is shown that any complete, non-flat, properly embedded minimal disk can be decomposed into two regions: one a region of strict spiraling, i.e., the union of two strictly spiraling multi-valued graphs over the $x_{3}=0$ plane (after a rotation of $\mathbb{R}^{3}$ ), and the other a neighborhood of the region where the graphs are joined and where the normal has small vertical component. By strictly spiraling, we mean that each sheet of the graph meets any (appropriately centered) cylinder with axis parallel to the $x_{3}$-axis in a curve along which $x_{3}$ strictly increases (or decreases). This follows from existence results for multi-valued minimal graphs in embedded disks found in [5] and an approximation result for such minimal graphs from [3]. The strict spiraling is then used to see that $\nabla_{\Sigma} x_{3} \neq 0$ everywhere on the surface; thus, the Gauss map is not vertical and the holomorphic map $z=x_{3}+i x_{3}^{*}$ is a holomorphic coordinate. By looking at the log of the stereographic projection of the Gauss map, the strict spiraling is used to show that $z$ is actually a proper map and thus, conformally, the surface is the plane. Finally, this gives strong rigidity for the Weierstrass data, implying the surface is a helicoid.

For $\Sigma \in \mathcal{E}(1,+)$, as there is finite genus and only one end, the topology of $\Sigma$ lies in a ball in $\mathbb{R}^{3}$, and so, by the maximum principle, all components of the intersection of $\Sigma$ with a ball disjoint from the genus are disks. Hence, outside of a large ball, one may use the local results of [4], [5], [6], [7] about embedded minimal disks. In [1], the trivial topology of $\Sigma$ allows one to deduce global geometric structure immediately from these local results. For $\Sigma \in \mathcal{E}(1,+)$, the presence of non-zero genus complicates matters. Nevertheless, the global structure will follow from the far reaching description of embedded minimal surfaces given by Colding and Minicozzi in [9]. In particular, as $\Sigma$ has one end, globally it looks like a helicoid (see Appendix D). Following [1], we first prove a sharper description of the global structure (in Section 3.2); indeed, one may generalize the decomposition of [1] to $\Sigma \in \mathcal{E}(1,+)$ as:

Theorem 1.5. There exist $\epsilon_{0}>0$ and a decomposition (see Figure 2) of $\Sigma$ into disjoint subsets $\mathcal{R}_{A}, \mathcal{R}_{S}$, and $\mathcal{R}_{G}$ such that:

(1) $\mathcal{R}_{G}$ is compact, connected, has connected boundary and $\Sigma \backslash \mathcal{R}_{G}$ has genus 0 ;

(2) after a rotation of $\mathbb{R}^{3}, \mathcal{R}_{S}$ can be written as the union of two (oppositely oriented) strictly spiraling multi-valued graphs $\Sigma^{1}$ and $\Sigma^{2}$; 
(3) in $\mathcal{R}_{A},\left|\nabla_{\Sigma} x_{3}\right| \geq \epsilon_{0}$.

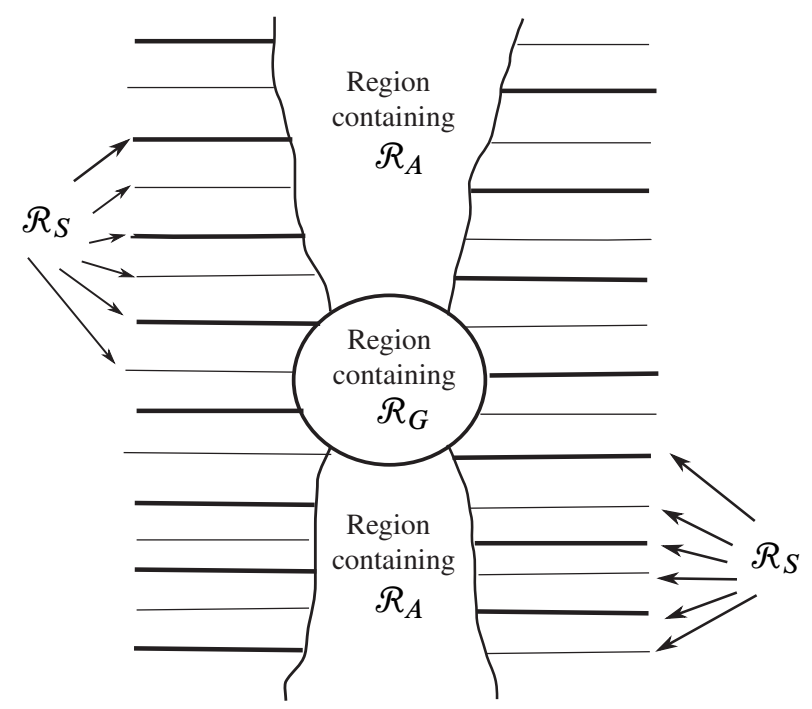

Figure 2. A cross-sectional sketch of the three regions in the decomposition of $\Sigma$ as outlined in Theorem 1.5.

Remark 1.6. We say $\Sigma^{i}(i=1,2)$ is a multi-valued graph if it can be decomposed into $N$-valued $\epsilon$-sheets (see Definition 2.2) with varying center. That is, $\Sigma^{i}=\bigcup_{j=-\infty}^{\infty} \Sigma_{j}^{i}$ where each $\Sigma_{j}^{i}=y_{j}^{i}+\Gamma_{u_{j}^{i}}$ is an $N$-valued $\epsilon$-sheet. Strict spiraling is then equivalent to $\left(u_{j}^{i}\right)_{\theta} \neq 0$ for all $j$. A priori, the axes of the multi-valued graphs vary, a fact that introduces additional book-keeping. For the sake of clarity, we assume that each $\Sigma^{i}$ is an $\infty$-valued $\epsilon$-sheet - i.e., $\Sigma^{i}$ is the graph, $\Gamma_{u^{i}}$, of a single function $u^{i}$ with $u_{\theta}^{i} \neq 0$.

To prove this decomposition, we first find the region of strict spiraling, $\mathcal{R}_{S}$. The strict spiraling controls the asymptotic behavior of level sets of $x_{3}$ which, as $x_{3}$ is harmonic on $\Sigma$, gives information about $x_{3}$ in all of $\Sigma$. More precisely:

Proposition 1.7. There exists $\Gamma \subset \Sigma$, an annulus, so that $\Sigma \backslash \Gamma$ is compact and such that $\nabla_{\Sigma} x_{3} \neq 0$ in $\Gamma$. Further, for all $c \in \mathbb{R}, \Gamma \cap\left\{x_{3}=c\right\}$ consists of either one smooth, properly embedded curve or two smooth, properly embedded curves each with one endpoint on $\partial \Gamma$ along with a finite number of smooth curves with both endpoints on $\partial \Gamma$. Moreover, if $c$ is a regular value of $x_{3}$ then $\Gamma \cap\left\{x_{3}=c\right\}$ is a subset of the unbounded component of $\Sigma \cap\left\{x_{3}=c\right\}$. 
The decomposition allows us to argue as in [1], though the non-trivial topology again adds some technical difficulties. By Stokes' Theorem, $x_{3}^{*}$ (the harmonic conjugate of $x_{3}$ ) exists on $\Gamma$ and thus there is a well defined holomorphic map $z: \Gamma \rightarrow \mathbb{C}$ given by $z=x_{3}+i x_{3}^{*}$. Proposition 1.7 implies that $z$ is a holomorphic coordinate on $\Gamma$. We claim that $z$ is actually a proper map and so $\Gamma$ is conformally a punctured disk. Following [1], this can be shown by studying the Gauss map. On $\Gamma$, the stereographic projection of the Gauss map, $g$, is a holomorphic map that avoids the origin. Moreover, the minimality of $\Sigma$ and the strict spiraling in $\mathcal{R}_{S}$ imply that the winding number of $g$ around the inner boundary of $\Gamma$ is zero. Hence, by monodromy there exists a holomorphic map $f: \Gamma \rightarrow \mathbb{C}$ with $g=e^{f}$. Then, as in [1], the strict spiraling in $\mathcal{R}_{S}$ imposes strong control on $f$ which is sufficient to show that $z$ is proper. Further, once we establish $\Gamma$ is conformally a punctured disk, the properties of the level sets of $f$ imply that it extends meromorphically over the puncture with a simple pole. This gives Theorem 1.1.

\section{Structural properties of $\Sigma$}

In the next five subsections, we develop the tools needed to prove the decomposition of Theorem 1.5 and Proposition 1.7. Many of these are extensions of those developed for the simply connected case, which can be found in Section 2 of [1].

2.1. Preliminaries. We first introduce some notation. Unless otherwise specified, throughout the paper let $\Sigma \in \mathcal{E}(1,+)$, i.e., $\Sigma$ is a complete, embedded minimal surface with finite and positive genus, $k$, and one end. Here we say that a surface has genus $k$ if it is homeomorphic to a compact, oriented genus $k$ surface with at most a finite number of punctures. As $\Sigma$ has one end and is complete in $\mathbb{R}^{3}$, there exists an $R>0$ so that one of the components $\bar{\Sigma}$ of $\Sigma \cap B_{R}$ is a compact surface with connected boundary and genus $k$; we refer to $\bar{\Sigma}$ as the genus of $\Sigma$. Thus, $\Sigma \backslash \bar{\Sigma}$ has genus 0 and is a neighborhood of the end of $\Sigma$. By homothetically rescaling, we may assume that the genus, $\bar{\Sigma}$, lies in $B_{1}$ and $\sup _{\bar{\Sigma}}|A|^{2} \geq 1$. Here $|A|^{2}$ denotes the norm squared of the second fundamental form of $\Sigma$ and $B_{r}(y)$ represents the Euclidean ball of radius $r$, centered at $y$, whereas $\mathscr{B}_{r}(y)$ denotes the intrinsic ball in $\Sigma$ of radius $r$ centered at $y$. Throughout the paper, when we say far from (or near) the genus, we mean extrinsically with respect to this scale. That is, a subset of $\mathbb{R}^{3}$ is far from the genus if the Euclidean distance to $B_{1}$ is large.

Denote by $\Pi: \mathbb{R}^{3} \rightarrow \mathbb{R}^{2}$ the projection $\Pi\left(x_{1}, x_{2}, x_{3}\right)=\left(x_{1}, x_{2}\right)$. Let

$$
\mathbf{C}_{\delta}(y)=\left\{x:\left(x_{3}-y_{3}\right)^{2} \leq \delta^{2}\left(\left(x_{1}-y_{1}\right)^{2}+\left(x_{2}-y_{2}\right)^{2}\right)\right\} \subset \mathbb{R}^{3}
$$

be the complement of a cone and set $\mathbf{C}_{\delta}=\mathbf{C}_{\delta}(0)$. Given a real-valued function, $u$, defined on a domain $\Omega \subset \mathbb{R}^{+} \times \mathbb{R}$, define the map $\Phi_{u}: \Omega \rightarrow \mathbb{R}^{3}$ by $\Phi_{u}(\rho, \theta)=$ 
( $\rho \cos \theta, \rho \sin \theta, u(\rho, \theta)$ ) so that the image is a multi-valued graph. A natural domain is the polar rectangle

$$
S_{r_{1}, r_{2}}^{\theta_{1}, \theta_{2}}=\left\{(\rho, \theta) \mid r_{1} \leq \rho \leq r_{2}, \theta_{1} \leq \theta \leq \theta_{2}\right\} .
$$

Indeed, for $u$ defined on $S_{r_{1}, r_{2}}^{\theta_{1}, \theta_{2}}, \Phi_{u}\left(S_{r_{1}, r_{2}}^{\theta_{1}, \theta_{2}}\right)$ is a multi-valued graph over the annulus $D_{r_{2}} \backslash D_{r_{1}}$. Thus, $\Gamma_{u}:=\Phi_{u}(\Omega)$ is the graph of $u$, and $\Gamma_{u}$ is embedded if and only if $w \neq 0$, where the separation $w$ of $u$ is defined as $w(\rho, \theta)=u(\rho, \theta+2 \pi)-u(\rho, \theta)$. We say a multi-valued graph, $\Gamma_{u}$, strictly spirals if, for $u: \Omega \rightarrow \mathbb{R}^{3}, u_{\theta} \neq 0$. Note at times we fail to distinguish between $u$ and its graph $\Gamma_{u}$ - the meaning will be clear from context.

Recall that $u$ satisfies the minimal surface equation if

$$
\operatorname{div}\left(\frac{\nabla u}{\sqrt{1+|\nabla u|^{2}}}\right)=0 .
$$

The graphs of interest to us will satisfy the following flatness condition:

$$
|\nabla u|+\rho\left|\operatorname{Hess}_{u}\right|+4 \rho \frac{|\nabla w|}{|w|}+\rho^{2} \frac{\left|\operatorname{Hess}_{w}\right|}{|w|} \leq \epsilon<\frac{1}{2 \pi} .
$$

As multi-valued minimal graphs are fundamental to the description of the asymptotic behavior, we introduce some notation for them.

Definition 2.1. A multi-valued minimal graph $\Sigma_{0}$ is a weak $N$-valued $(\epsilon$-)sheet (centered at $y$ on the scale $s$ ), if $\Sigma_{0}=\Gamma_{u}+y$ and $u$, defined on $S_{s, \infty}^{-\pi N, \pi N}$, satisfies (2.3), has $|\nabla u| \leq \epsilon$, and $\Sigma_{0} \subset \mathbf{C}_{\epsilon}(y)$.

We will often need more control on the sheets as well as a normalization at $\infty$ :

Definition 2.2. A multi-valued minimal graph $\Sigma_{0}$ is an (strong) $N$-valued $(\epsilon$-)sheet (centered at $y$ on the scale s), if $\Sigma_{0}=\Gamma_{u}+y$ is a weak $N$-valued $\epsilon$-sheet centered at $y$ on scale $s$, and in addition $u$ satisfies (2.4) and $\lim _{\rho \rightarrow \infty} \nabla u(\rho, 0)=0$.

Using Simons' inequality, Corollary 2.3 of [2] shows that on the one-valued middle sheet of a 2-valued graph satisfying (2.4), the hessian of $u$ has faster than linear decay. This implies a Bers like result on asymptotic tangent planes - i.e., the normalization at $\infty$ in the definition of $\epsilon$-sheet is well defined. Indeed, for $\Gamma_{u}$ a 2-valued $\epsilon$-sheet, one has gradient decay,

$$
|\nabla u|(\rho, 0) \leq C \epsilon \rho^{-5 / 12} .
$$

Note that Colding and Minicozzi show, using standard elliptic theory, that for sufficiently large $N$ and small $\delta$ a weak $N$-valued $\delta$-sheet contains a 4 -valued $\epsilon$ sheet as a sub-graph. For the details, we refer the reader to Proposition 2.3 of [1]. 
Finally, as in the papers of Colding and Minicozzi, we are interested in points with large curvature relative to nearby points, as around these points multi-valued graphs form (see [5]). The precise definition we use is the following:

Definition 2.3. The pair $(y, s), y \in \Sigma, s>0$, is a $(C)$ blow-up pair on scale $s$ if

$$
\sup _{B_{s}(y) \cap \Sigma}|A|^{2} \leq 4|A|^{2}(y)=4 C^{2} s^{-2} .
$$

Remark 2.4. The constant $C$ will be specified in some of the theorems but it should always be thought of as being very large.

2.2. Existence of multi-valued graphs. To obtain the decomposition of Theorem 1.5 we will need two propositions regarding the large scale geometric structure of elements of $\mathcal{E}(1,+)$. These generalize results for disks from [4] and [5] on the existence and extendability of multi-valued graphs in embedded minimal disks. It should be noted that many of the proofs of [4], [5] did not require that the surface be a disk but only that the boundary be connected, a fact used in [9] to extend the description of embedded minimal disks of [4], [5] to finite genus surfaces. The first result we will need gives the existence of an $N$-valued graph starting near the genus and extending as a graph all the way out; i.e., the initial graph is a subset of a graph over an unbounded annulus. The second result is similar but applies for a blow-up pair far from the genus. Namely, for such a pair a multi-valued graph forms on the scale of the pair and extends as a graph all the way out. It may be helpful to compare with the analogous results for disks, i.e. Theorem 0.3 of [4] and Theorem 0.4 of [5].

Note that local versions of the propositions stated below are used by Colding and Minicozzi in [9], specifically in the proof of their compactness result for finite genus surfaces (i.e. Theorem 0.9 of [9]). However, they are not explicitly stated in [9]. Thus, for the sake of completeness, we include a proof of both propositions, assuming Theorem 0.9 of [9], in Appendix D. Both propositions require a rotation of $\mathbb{R}^{3}$. However, because both propositions come from the global geometric structure of $\Sigma$, the rotations are the same.

Proposition 2.5. Given $\epsilon>0$ and $N \in \mathbb{Z}^{+}$there exists an $R>1$ so that the following holds. After a rotation of $\mathbb{R}^{3}$ there exists $\Sigma_{g} \subset \Sigma$, a weak $N$-valued $\epsilon$-sheet centered at 0 and on scale $R$.

Proposition 2.6. Given $\epsilon>0$ sufficiently small and $N \in \mathbb{Z}^{+}$there exist $C_{1}>0$ and $R>1$ so that the following holds. After a rotation of $\mathbb{R}^{3}$, if $(y, s)$ is a $C_{1}$ blow-up pair in $\Sigma$ and $|y| \geq R$ then there exists $\Sigma_{g} \subset \Sigma$, a weak $N$-valued $\epsilon$-sheet centered at $y$ and on scale $s$. 
2.3. Global structure of $\Sigma$. For an element $\Sigma \in \mathcal{E}(1,0)$ (i.e. a minimal disk), the existence of a weak $\epsilon$-sheet (i.e. the existence of a blow-up pair) allowed one to immediately appeal to the one-sided curvature estimates of [7] (see Appendix C). This allowed one to deduce important information about the global structure of $\Sigma$. In particular, one had a type of "regularity" for the set of blow-up pairs; that is, all the blow-up pairs of $\Sigma$ were forced to lie within a wide cone. A related property was that if $(0,1)$ is a blow-up pair in $\Sigma$ then blow-up pairs far from 0 have scale a small fraction of the distance to 0 .

We will need a similar results for $\Sigma \in \mathcal{E}(1,+)$; however, because the one-sided curvature estimate is very sensitive to the topology, the non-trivial genus will introduce some technical difficulties. We discuss how to overcome these in Appendix C. As a consequence, we have the following lemma, which asserts that there is a cone (centered at the origin) so that for blow-up pairs far from the genus, the pair must lie within the cone, i.e., we recover the "regularity" of the set of blow-up pairs. We point out that this result is particularly useful when combined with Corollary C.3 and Proposition 2.6, as the three imply that for small $\delta^{\prime}$ and a blow-up pair $(y, s)$ far from the genus, one may apply the one-sided curvature estimate in $\mathbf{C}_{\delta^{\prime}}(y)$ exactly as was done in the case for disks.

Lemma 2.7. There exists $a \delta>0$ and $R>1$ so that if $(y, s)$ is a blow-up pair in $\Sigma$ and $|y| \geq R$ then $y \notin \mathbf{C}_{\delta}(0)$.

Proof. Fix $\epsilon=1 / 2$, and let $\delta_{0}$ be the value given by Corollary C.2. By Proposition 2.5, $\Sigma$ contains a weak 2-valued $\delta_{0}$-sheet centered at 0 and with scale $R_{0}>1$. Thus, as $\bar{\Sigma} \subset B_{1} \subset B_{R_{0}}$, we may apply Corollary C.2 to deduce that in the set $\mathbf{C}_{\delta_{0}} \backslash B_{2 R_{0}}$ every component of $\Sigma$ is a graph with gradient bounded by $1 / 2$. In particular, there are no blow-up pairs in this set. Thus, we may take $R=2 R_{0}$ and $\delta=\delta_{0}$.

The second global result for disks also generalizes. Indeed, we claim that the further a blow-up pair is from the genus, the smaller the ratio between the scale and the distance to the genus. This is an immediate consequence of the control on curvature around blow-up pairs as given by Proposition B.1 (an extension of Lemma 2.26 of [8] to $\Sigma$ ). Indeed, for blow-up pairs far from the genus, the scale is small relative to distance to the genus:

Corollary 2.8. Given $\alpha, C_{1}>0$ there exists an $R$ such that if $(y, s)$ is a $C_{1}$ blow-up pair of $\Sigma$ with $|y| \geq R$ then $s<\alpha|y|$.

Proof. Recall that we have normalized $\Sigma$ so that $\sup _{B_{1} \cap \Sigma}|A|^{2} \geq 1$. Now suppose the result did not hold. Then there exists a sequence $\left(y_{j}, s_{j}\right)$ of $C_{1}$ blow-up pairs with $\left|y_{j}\right| \geq j$ and $s_{j} \geq \alpha\left|y_{j}\right|$. Set $K_{1}=2 / \alpha$. By Proposition B. 1 there exists $K_{2}$ such that 
$\sup _{B_{K_{1} s_{j}}\left(y_{j}\right) \cap \Sigma}|A|^{2} \leq K_{2} s_{j}^{-2}$. Since $B_{1} \subset B_{K_{1} s_{j}}\left(y_{j}\right), \sup _{B_{1} \cap \Sigma}|A|^{2} \leq K_{2} s_{j}^{-2}$. But $s_{j} \geq \alpha\left|y_{j}\right| \geq \alpha j$; thus for $j$ sufficiently large one obtains a contradiction.

2.4. Blow-up sheets. In order to get the strict spiraling in the decomposition of Theorem 1.5, we need to check that the multi-valued graphs that make up most of $\Sigma$ can be consistently normalized. To that end, we note that, for blow-up pairs far enough from the genus, one obtains a nearby $\epsilon$-sheet (i.e., we have a normalized multi-valued graph). This is essentially Theorem 2.5 in [1].

Theorem 2.9. Given $\epsilon>0, N \in \mathbb{Z}^{+}$, there exist $C_{1}, C_{2}>0$ and $R>0$ such that the following holds true. Suppose that $(y, s)$ is a $C_{1}$ blow-up pair of $\Sigma$ with $|y|>R$. Then there exists (after a rotation of $\mathbb{R}^{3}$ ) an $N$-valued $\epsilon$-sheet $\Sigma_{1}=y+\Gamma_{u_{1}}$ centered at $y$ on scale s. Moreover, the separation over $\partial D_{s}(\Pi(y))$ of $\Sigma_{1}$ is bounded below by $C_{2} s$.

The proof of the theorem is exactly the same as the proof of Theorem 2.5 in [1] with one modification. Where the proof of [1] uses Theorem 0.2 of [5] to produce a weak $N_{0}$-valued sheet ( $N_{0}$ is determined in the proof), one must now use Proposition 2.6. Thus, in the above hypothesis, the blow-up pair must satisfy the additional criteria of $|y|>R$ so that one may appeal to Proposition 2.6.

Following Colding and Minicozzi, we need to next understand the structure of $\Sigma$ between the sheets of this initial multi-valued graph, $\Sigma_{1}$. We claim that in between this sheet, $\Sigma$ consists of exactly one other $\epsilon$-sheet. To make this more precise, suppose $u$ is defined on $S_{1 / 2, \infty}^{-\pi N-3 \pi, \pi N+3 \pi}$ and $\Gamma_{u}$ is embedded. We define $E$ to be the region over $D_{\infty} \backslash D_{1}$ between the top and bottom sheets of the concentric sub-graph of $u$. That is:

$$
\begin{aligned}
E=\{(\rho \cos \theta, \rho \sin \theta, t): & 1 \leq \rho \leq \infty,-2 \pi \leq \theta<0, \\
& u(\rho, \theta-\pi N)<t<u(\rho, \theta+(N+2) \pi\} .
\end{aligned}
$$

When $\Sigma$ is a disk, Colding and Minicozzi in Theorem I.0.10 of [7] show that $\Sigma \cap E \backslash \Sigma_{1}$ consists of a single graphical piece. Thus, using $\Sigma_{1}$ and the one-sided curvature estimate of [7], the gradient of this second graphical component is controlled. As before, when there are enough sheets in this second multi-valued graph and the gradient is controlled, standard elliptic theory establishes (2.4) on a sub-graph and hence one obtains two $\epsilon$-sheets spiraling together. We refer the reader to Theorem 2.6 of [1] for the details. In the more general setting of this paper, as long as the part of $\Sigma$ between the sheets of $\Sigma_{1}$ makes up a second minimal graph and we can apply the one-sided curvature estimates, the proof of Theorem 2.6 of [1] applies. Thus, we must verify both the existence of this second multi-valued graph and that we are able to apply the one-sided curvature estimate to it. By patching together two results of Colding and Minicozzi from [7] the first issue is easily handled. The global structure 
of $\Sigma$, in particular Lemma 2.7, implies that as long as the blow-up pair is far from the genus there is no problem handling the second issue either:

Theorem 2.10. Given $\epsilon>0$ sufficiently small there exist $C_{1}, C_{2}>0$ and $R>1$ so that the following holds. Suppose $(y, s)$ is a $C_{1}$ blow-up pair, with $|y|>R$. Then there exist two 4-valued $\epsilon$-sheets $\Sigma_{i}=y+\Gamma_{u_{i}}(i=1,2)$ on the scale $s$ centered at $y$ which spiral together (i.e. $u_{1}(s, 0)<u_{2}(s, 0)<u_{1}(s, 2 \pi)$ ). Moreover, the separation over $\partial D_{s}(\Pi(y))$ of $\Sigma_{i}$ is bounded below by $C_{2} s$.

Remark 2.11. We refer to $\Sigma_{1}, \Sigma_{2}$ as ( $\epsilon$-)blow-up sheets associated with $(y, s)$.

Proof. Let $\delta>0$ and $R>1$ be given by Lemma 2.7. Using this $\delta$ and $\epsilon / 2$, pick $\delta_{0}<\epsilon / 2$ as in Corollary C.3 (and increase $R$ if needed). Theorem 2.9 gives one $\widetilde{N}$-valued $\delta_{0}$-sheet, $\Sigma_{1}$, forming near $(y, s)$ for appropriately chosen $C_{1}$ (and possibly after again increasing $R$ ). Here we choose the $\widetilde{N}>4$ as in Theorem 2.6 of [1] this allows one to establish (2.4) on a sub-graph of the second graph. Indeed, once we establish that $E$, the region between the sheets of $\Sigma_{1}$, is a weak $(\tilde{N}-4)$-valued $\epsilon / 2$-sheet the argument of Theorem 2.6 of [1] carries over unchanged.

We now show that $\Sigma \cap E \backslash \Sigma_{1}$ consists of exactly one multi-valued graph. Theorem I.0.10 of [7] implies that near the blow-up pair the part of $\Sigma$ between $\Sigma_{1}$ is a $\tilde{N}-4$ sheeted graph $\Sigma_{2}^{\text {in }}$; i.e. if $R_{0}$ is chosen so that $B_{4 R_{0}}(y)$ is disjoint from the genus then $B_{R_{0}}(y) \cap E \cap \Sigma \backslash \Sigma_{1}=\Sigma_{2}^{\text {in }}$. To ensure $\Sigma_{2}^{\text {in }}$ is non-empty, we increase $R$ so that $|y| \geq 8 s$ (which we may do by Corollary 2.8). On the other hand, Appendix D of [7] guarantees that, outside of a very large ball centered at the genus, the part of $\Sigma$ between $\Sigma_{1}$ is a $\widetilde{N}-4$ sheeted graph, $\Sigma_{2}^{\text {out }}$. That is, for $R_{1} \geq|y|$ large, $E \cap \Sigma \backslash\left(B_{R_{1}} \cup \Sigma_{1}\right)=\Sigma_{2}^{\text {out }}$. By our choice of $\delta_{0}$, $R$, we can now apply the one-sided curvature estimate in $E$, and so all the components of $E \backslash \Sigma_{1}$ are graphs with gradient bounded by $\epsilon / 2$. Thus, it suffices to show that $\Sigma_{2}^{\text {in }}$ and $\Sigma_{2}^{\text {out }}$ are subsets of the same component. If this was not the case, then, as $\Sigma_{2}^{\text {in }}$ is a graph and $\Sigma$ is complete, $\Sigma_{2}^{\text {in }}$ must extend inside $E$ beyond $B_{R_{1}}$. But this contradicts Appendix D of [7] by giving two components of $\Sigma \backslash \Sigma_{1}$ in $E \cap \Sigma \backslash B_{R_{1}}$.

2.5. Existence of blow-up pairs. While the properties of $\epsilon$-sheets will give the strictly spiraling region of $\Sigma, \mathcal{R}_{S}$, to understand the region where these sheets fit together (i.e. what will become $\mathcal{R}_{A}$ ), we need a handle on the distribution of the blow-up pairs of $\Sigma$. Notice that the global structural results discussed above, i.e. Lemma 2.7 and Corollary 2.8, give weak information of this sort.

In the case of trivial topology - i.e. Theorem 2.8 of [1] - non-flatness gives one blow-up pair $\left(y_{0}, s_{0}\right)$, which in turn yields associated blow-up sheets. Then by Corollary III.3.5 of [6] coupled with the one-sided curvature estimate, the blow-up sheets give the existence of nearby blow-up pairs $\left(y_{ \pm 1}, s_{ \pm 1}\right)$ above and below $\left(y_{0}, s_{0}\right)$ 
(see also Lemma 2.5 of [8]). Iterating, one determines a sequence of blow-up pairs that are then used to construct the decomposition. The extension of the argument to surfaces in $\mathscr{E}(1,+)$ is much the same, though again there are various technical difficulties complicating matters. Essentially, the proof will rely on three things. First, the result of [6] is local; it depends on the topology being trivial in a large ball relative to the scale of the blow-up pair. Second, by Lemma 2.7 and Corollary C.3, for blow-up pairs sufficiently far from the genus, we can apply the one-sided curvature estimate. Thus, we conclude that points of large curvature near a blow-up pair must lie within a cone with vertex the point of the blow-up pair. As a consequence, blow-up pairs can be constructed that are truly above (or below) a given blow-up pair. Third, Corollary 2.8 implies that the scale of blow-up pairs far from the genus is small relative to this distance.

Thus, it will suffice to find two blow-up pairs far from the genus in $\Sigma$, one above and one below the genus. We first verify this is possible:

Lemma 2.12. Given $\epsilon>0$ sufficiently small and $h>1, C_{1}>0$, there exist pairs $\left(y_{ \pm}, s_{ \pm}\right)$such that $\left(y_{ \pm}, s_{ \pm}\right)$are $C_{1}$ blow-up pairs of $\Sigma$ and $x_{3}\left(y_{+}\right)>h>-h>$ $x_{3}\left(y_{-}\right)$.

Proof. Fix a $\delta_{0}>0$ small, it will be specified in what follows. Proposition 2.5 of this paper and Appendix D of [7] together guarantee the existence of two $\widetilde{N}$-valued graphs spiraling together over an unbounded annulus (with inner radius $\bar{R}$ ) and lying in $\mathbf{C}_{\delta_{0}}$. Moreover, by construction, one of these is a weak $\delta_{0}$-sheet. By using Corollary C.2, and replacing $\bar{R}$ by $2 \bar{R}$ we can control the gradient on both $\widetilde{N}$-valued graphs. As before, for large enough $\widetilde{N}$ and sufficiently small $\delta_{0}$, we get (2.4) on a sub-graph of both graphs. Because Proposition 2.5 already provides the necessary rotation, we get two $N$-valued $\epsilon$-sheets around the genus, $\Sigma_{1}, \Sigma_{2}$, on some scale $\widetilde{R}$ and in $\mathbf{C}_{\epsilon}$. We may make $\epsilon$ as small as we like by shrinking $\delta_{0}$.

Theorem III.3.1 of [6] is the analogue to Corollary III.3.5 of [6] for minimal surfaces with connected boundary. Thus, for any $r_{0} \geq \max \{1, \widetilde{R}\}$, Theorem III.3.1 implies there is large curvature above and below the genus at points $x_{ \pm}$. Precisely, there exist $x_{ \pm} \in \Sigma \backslash B_{4 r_{0}}$ such that $\left|x_{ \pm}\right|^{2}|A|^{2}\left(x_{ \pm}\right) \geq 4 C_{1}^{2}$. Hence, by a standard blow-up argument (see Lemma 5.1 of [5]), one gets the desired $C_{1}$ blow-up pairs $\left(y_{ \pm}, s_{ \pm}\right)$above and below the genus and with $\left|y_{ \pm}\right| \geq \gamma r_{0}$ where here $\gamma$ is small and depends only on $C_{1}$. Lemma 2.7 implies, after increasing $r_{0}$ if needed, that the $y_{ \pm}$ do not lie in $\mathbf{C}_{\delta}(0)$ and thus by increasing $r_{0}$ further (by an amount depending only on $\gamma, \delta$ and $h)$ one has $x_{3}\left(y_{+}\right)>h>-h>x_{3}\left(y_{-}\right)$.

Thus, we may iteratively construct the desired sequence of blow-up pairs. This sequence will be used to construct the region $\mathcal{R}_{A}$ in the next section. 
Proposition 2.13. Given $\epsilon>0$ sufficiently small, there exist constants $C_{1}, C_{\text {in }}>0$ and a sequence $\left(\tilde{y}_{i}, \tilde{s}_{i}\right)(i \in \mathbb{Z} \backslash\{0\})$ of $C_{1}$ blow-up pairs of $\Sigma$ such that the sheets associated to $\left(\tilde{y}_{i}, \tilde{s}_{i}\right)$ are $\epsilon$-sheets on scale $\tilde{s}_{i}$ centered at $\tilde{y}_{i}$ and $x_{3}\left(\tilde{y}_{i}\right)<x_{3}\left(\tilde{y}_{i+1}\right)$. Moreover, for $i \geq 1, \tilde{y}_{i+1} \in B_{C_{\mathrm{in}} \tilde{s}_{i}}\left(\tilde{y}_{i}\right)$ while for $i \leq-1, \tilde{y}_{i-1} \in B_{C_{\mathrm{in}} \tilde{s}_{i}}\left(\tilde{y}_{i}\right)$.

Proof. Without loss of generality, we work above the genus (i.e. for $x_{3}>1$ and $i \geq 1$ ), as the argument below the genus is identical. Let $\delta, R>0$ be given by Lemma 2.7. Thus, if $(y, s)$ is a blow-up pair in $\Sigma$ so that $|y| \geq R$ then $y \notin \mathbf{C}_{\delta}$. Moreover, using $\epsilon$ and $\delta$, let $\delta_{0}$ be given by Corollary C.3 and increase, if needed, $R$ as indicated by the corollary. We are free to shrink $\delta_{0}$, so assume that $\delta_{0} \leq \epsilon$. Use Theorem 2.10 with $\delta_{0}$ to choose $C_{1}, C_{2}$ and increase $R$, if needed, as indicated by the theorem. Thus, for any $(y, s)$ a $C_{1}$ blow-up pair with $|y| \geq R$, we have $\delta_{0}$-sheets (which, as $\delta_{0} \leq \epsilon$ are also $\epsilon$-sheets) associated to $(y, s)$. Moreover, this and the choice of $R$ imply that Corollary C.3 applies in $\mathbf{C}_{\delta_{0}}(y) \backslash B_{2 s}(y)$.

Corollary III.3.5 of [6] and a standard blow-up argument give constants $C_{\text {out }}>$ $C_{\text {in }}>0$ such that, for a $C_{1}$ blow-up pair $(y, s)$, as long as the component of $B_{C_{\text {out }}}(y) \cap$ $\Sigma$ containing $y$ is a disk and there are blow-up sheets associated to $(y, s)$, then we can find blow-up pairs $\left(y_{ \pm}, s_{ \pm}\right)$above and below $(y, s)$ (in a weak sense) and inside $B_{C_{\text {in }}}(y)$. If, in addition, we can apply Corollary C.3 centered at $y$, then we can ensure $x_{3}\left(y_{+}\right)>x_{3}(y)>x_{3}\left(y_{-}\right)$. Corollary 2.8 and Proposition A.1 together give a value $h_{1} \geq R$, depending on $C_{\text {out }}$, so for $|y| \geq h_{1}$ the component of $B_{C_{\text {out }} s}(y) \cap \Sigma$ containing $y$ is a disk.

It now suffices to find an initial blow-up pair $\left(\tilde{y}_{1}, \tilde{s}_{1}\right)$ with $x_{3}\left(\tilde{y}_{1}\right) \geq h_{1}$, as repeated application of the argument of the above paragraph gives the sequence $\left(\tilde{y}_{i}, \tilde{s}_{i}\right)$. Lemma 2.12, with $h_{1}$ replacing $h$, gives the existence of the desired initial blow-up pair.

\section{Structural decomposition of $\Sigma$}

We prove Theorem 1.5 and Proposition 1.7 in Subsection 3.2.

3.1. Constructing $\boldsymbol{R}_{S}$. The decomposition of $\Sigma$ now proceeds as in Section 4 of [1], with Proposition 3.1 giving strict spiraling far enough out in the $\epsilon$-sheets of $\Sigma$. After specifying a region of strict spiraling, $\mathcal{R}_{S}$, the remainder of $\Sigma$ will be split into the connected component containing the genus, $\mathcal{R}_{G}$, and the region containing the points of large curvature, $\mathcal{R}_{A}$.

In the interest of clarity we restate two results from [1] that we will need to prove our decomposition. The first result gives the strict spiraling of $\epsilon$-sheets.

Proposition 3.1 (Proposition 3.3 in [1]). There exists an $\epsilon_{0}$ so that the following holds. Suppose $\Gamma_{u}$ is a 3-valued $\epsilon$-sheet on scale 1 with $\epsilon<\epsilon_{0}$ and $w(1, \theta)=$ 
$u(1, \theta+2 \pi)-u(1, \theta) \geq C_{2}>0$. Then there exists $C_{3}=C_{3}\left(C_{2}\right) \geq 2$, so that on $S_{C_{3}, \infty}^{-\pi, \pi}$ we have

$$
u_{\theta}(\rho, \theta) \geq \frac{C_{2}}{8 \pi} \rho^{-\epsilon}
$$

The second result is a technical lemma that will guarantee that any sheets lying between sheets associated to consecutive blow-up pairs are eventually (for large enough radius) $\epsilon$-sheets. Results along these lines can by found in Section 5 of [2] and Section II.3 of [6] . Importantly, the proof of such a statement relies only on standard elliptic theory and the ability to apply the one-sided curvature estimate in $\mathbf{C}_{\delta_{1}}(y)$ for an appropriately chosen $\delta_{1}$, where $y \in \Sigma$ is the point of a blow-up pair. Lemma 2.7 and Corollary C.3 ensure, as long as we work far enough from the genus, that this last condition is satisfied. In order to avoid technicalities, we restrict attention only to pairs $\left(\tilde{y}_{i}, \tilde{s}_{i}\right)$ from Proposition 2.13 .

Lemma 3.2. There exists $\epsilon_{0}>0$ such that for given $N>4$ and $\epsilon_{0}>\epsilon>0$ there exists $R_{2}=R_{2}(\epsilon, N)>1$ so that if, using $\epsilon,\left(\tilde{y}_{i}, \tilde{s}_{i}\right)$ is a blow-up pair from Proposition 2.13 with two associated 4-valued $\epsilon$-sheets $\Sigma_{j}, j=1,2$, then there exist two $N$-valued $\epsilon$-sheets on scale $R_{2} \tilde{s}_{i}, \widetilde{\Sigma}_{j} \subset \Sigma$. Moreover, $\widetilde{\Sigma}_{j}$ may be chosen so that its 4-valued middle sheet contains $\Sigma_{j} \backslash\left\{\left(x_{1}-x_{1}\left(\tilde{y}_{i}\right)\right)^{2}+\left(x_{2}-x_{2}\left(\tilde{y}_{i}\right)\right)^{2} \leq R_{2}^{2} \tilde{s}_{i}^{2}\right\}$.

This is essentially Lemma 4.2 of [1], though the statement there is technically simpler. As before, the only obstruction to generalizing the proof from [1] is the possibility that we cannot apply the one-sided curvature estimates in $\mathbf{C}_{\delta}\left(\tilde{y}_{i}\right)$ for some small $\delta$. However, our choice of $\tilde{y}_{i}$ ensures this is not a problem.

We now wish to argue as in Lemma 4.3 of [1] (where we determine the regions $\mathcal{R}_{A}$ and $\mathcal{R}_{S}$ for disks). To do so we must ensure that we may use the chord-arc bounds of [8] near the pairs $\left(\tilde{y}_{i}, \tilde{s}_{i}\right)$. By choosing a subsequence of blow-up pairs $\left(y_{i}, s_{i}\right)$ that satisfy this additional criteria, we obtain the following:

Lemma 3.3. There exist constants $C_{1}, R_{0}, R_{1}$ and a sequence $\left(y_{i}, s_{i}\right)(i \neq 0)$ of $C_{1}$ blow-up pairs of $\Sigma$ so that $x_{3}\left(y_{i}\right)<x_{3}\left(y_{i+1}\right)$, and for $i \geq 1, y_{i+1} \in B_{R_{1} s_{i}}\left(y_{i}\right)$ while for $i \leq-1, y_{i-1} \in B_{R_{1} s_{i}}\left(y_{i}\right)$. Moreover, setting $\widetilde{\mathcal{R}}_{A}=\widetilde{\mathcal{R}}_{A}^{+} \cup \widetilde{\mathcal{R}}_{A}^{-}$, where $\widetilde{\mathcal{R}}_{A}^{ \pm}$ is the component of $\bigcup_{ \pm i>0} \Sigma \cap B_{R_{1} s_{i}}\left(y_{i}\right)$ containing $y_{ \pm 1}$, then $\Sigma \backslash\left(\widetilde{\mathcal{R}}_{A} \cup B_{R_{0}}\right)$ has exactly two unbounded components $\Sigma^{1}$ and $\Sigma^{2}$, each of which are strictly spiraling multi-valued graphs. We define the set $\widetilde{\mathcal{R}}_{S}=\Sigma^{1} \cup \Sigma^{2}$.

Proof. Fix $\epsilon<\epsilon_{0}$ where $\epsilon_{0}$ is smaller than the constants given by Proposition 3.1 and Lemma 3.2. Using this $\epsilon$, let $\left(\tilde{y}_{i}, \tilde{s}_{i}\right)$ be the sequence constructed in Lemma 2.13. Let us now determine how to choose the sequence $\left(y_{i}, s_{i}\right)$.

On $\left(y_{i}, s_{i}\right)$, we will need a uniform bound, $N$, on the number of sheets between the blow-up sheets associated to the pairs $\left(y_{i}, s_{i}\right)$ and $\left(y_{i+1}, s_{i+1}\right)$. This is equivalent 
to a uniform area bound which in turn follows from the uniform curvature bounds of Proposition B.1 of the appendix, once we can establish the appropriate chord-arc bounds. The proof of this is straightforward and can be found in Lemma 4.1 of [1]. Recall from [8] that the (strong) chord-arc bounds for minimal disks give a uniform constant $\beta>1$, so for any $r$, if the component of $B_{2(r+1) \beta s_{i}}\left(y_{i}\right) \cap \Sigma$ containing $y_{i}$ is a disk, then $B_{r s_{i}}\left(y_{i}\right) \cap \Sigma$ is a subset of $\mathcal{B}_{(r+1) \beta s_{i}}\left(y_{i}\right)$. To correctly apply the argument of Lemma 4.1 in [1], one must be sufficiently far from the genus; i.e. for a fixed constant $C_{\text {bnd }}$, the component of $B_{C_{\text {bnd }} s_{i}}\left(y_{i}\right) \cap \Sigma$ containing $y_{i}$ must be a disk. Note that $C_{\text {bnd }}$ depends only on $\beta$ and $C_{\text {in }}$ (where $C_{\text {in }}$ is as in Proposition 2.13). To that end, pick $h_{2} \geq 0$ by using Corollary 2.8 with $\alpha^{-1} \geq \max \left\{C_{\text {bnd }}, 2 \beta\left(R_{1}+1\right)\right\}$ where $R_{1}$ is to be chosen later. We then pick the sequence $\left(y_{i}, s_{i}\right)$ from $\left(\tilde{y}_{i}, \tilde{s}_{i}\right)$ by requiring $\left|x_{3}\left(y_{i}\right)\right| \geq h_{2}$ (and then relabeling). Notice that our method of choosing the $\left(y_{i}, s_{i}\right)$ ensures that $N$ is independent of our ultimate choice of $R_{1}$.

We now determine $R_{1}$ (see Figure 3). By choice of $\left(y_{i}, s_{i}\right)$, we can apply Lemma 3.2, so there exists an $R_{2}$ such that all of the (at most) $N$ sheets between the blow-up sheets associated to $\left(y_{1}, s_{1}\right)$ and $\left(y_{2}, s_{2}\right)$ are $\epsilon$-sheets on scale $R_{2} s_{1}$ centered on the line $\ell$ which goes through $y_{1}$ and is parallel to the $x_{3}$-axis. Label these

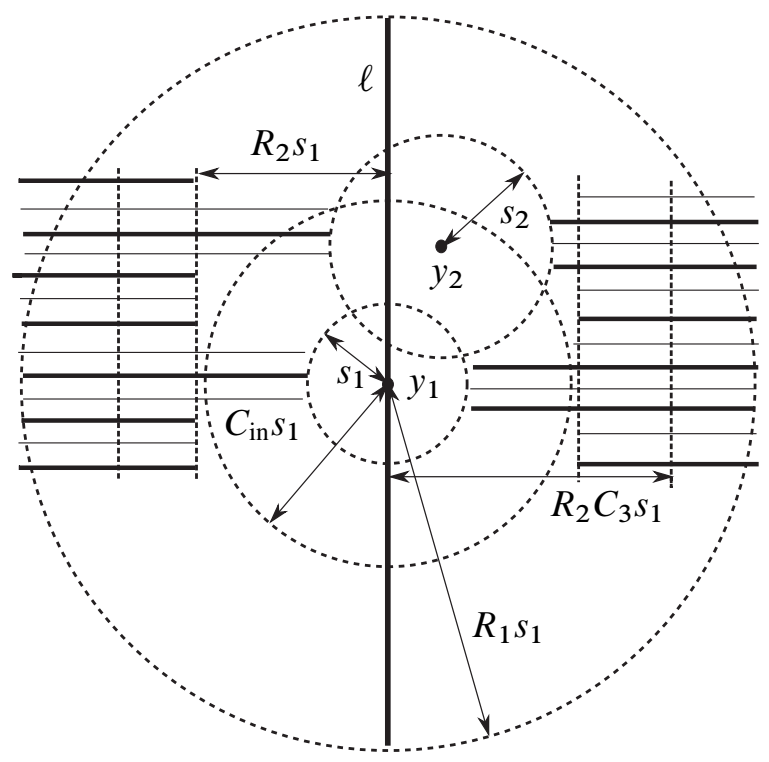

Figure 3. An illustration of the proof of Lemma 3.3.

pairs of $\epsilon$-sheets $\Sigma_{j}^{k}, k=1,2$ and $1 \leq j \leq N$. Integrating (2.4), and using $N$ and $C_{2}$ we get $\widetilde{C}_{2}$, so $\widetilde{C}_{2} s_{1}$ is a lower bound on the separation of each $\Sigma_{j}^{k}$ over the circle $\partial D_{R_{2} s_{1}}\left(\Pi\left(y_{1}\right)\right) \subset\left\{x_{3}=0\right\}$. Theorem 3.1 gives a $C_{3}$, depending on $\widetilde{C}_{2}$, such 
that outside of a cylinder centered at $\ell$ of radius $R_{2} C_{3} s_{1}$, all the $\Sigma_{j}^{k}$ strictly spiral. Choose $R_{1}$, depending only on $C_{\text {in }}, N, \epsilon, C_{3}, \beta$ and $R_{2}$, so that the component of $B_{R_{1} s_{1}}\left(y_{1}\right) \cap \Sigma$ containing $y_{1}$ also contains the point $y_{2}$ and the intersection of this cylinder with each $\Sigma_{j}^{k}$. This $R_{1}$ exists by the chord-arc bounds which we have by the choice of $\left(y_{i}, s_{i}\right)$. As there was nothing special about the blow-up pair $\left(y_{1}, s_{1}\right)$ in this argument and our conclusions are invariant under a rescaling, we can apply the same argument to each $\left(y_{i}, s_{i}\right)$ and thus construct $\widetilde{\mathcal{R}}_{A}$.

Finally, by properness, there exists a finite number, $M$, of $\epsilon$-sheets between the blow-up sheets associated to $\left(y_{ \pm 1}, s_{ \pm 1}\right)$. Pick $R_{0}$ large enough so that outside of the ball of radius $R_{0}$ the $M$ sheets between the blow-up sheets associated to $\left(y_{1}, s_{1}\right)$ and $\left(y_{-1}, s_{-1}\right)$ strictly spiral. Such an $R_{0}$ exists by Proposition 2.5 , Theorem 3.2, and the above argument. By the above construction, the $\Sigma^{i}$ are strictly spiraling multi-valued graphs as described in Remark 1.6.

Notice that $\Sigma$ is not necessarily contained in $\widetilde{\mathcal{R}}_{A} \cup \widetilde{\mathcal{R}}_{S} \cup B_{R_{0}}$. In the next section, we will adjust these subsets in order to obtain the decomposition.

3.2. Decomposing $\Sigma$. The strict spiraling, the fact that away from the genus convex sets meet $\Sigma$ in disks (see Lemma A.1) and the proof of Rado's theorem (see [24], [25]) will give $\nabla_{\Sigma} x_{3} \neq 0$ in $\mathcal{R}_{A}$. Then a Harnack inequality will allow us to bound $\left|\nabla_{\Sigma} x_{3}\right|$ from below on $\mathcal{R}_{A}$. We first use the strict spiraling on $\widetilde{R}_{S}$ and an appropriate initial choice for $\mathcal{R}_{G}$ to determine the behavior of the level sets of $x_{3}$.

Proof of Proposition 1.7. By the properness of $\Sigma$ there exists an $R_{0}^{\prime} \geq R_{0}$, where $R_{0}$ is from Lemma 3.3, so that the component of $B_{R_{0}^{\prime}} \cap \Sigma$ containing $\bar{\Sigma}$ contains $B_{R_{0}} \cap \Sigma$. We take $\mathcal{R}_{G}$ to be this component and define $\Gamma=\Sigma \backslash \mathcal{R}_{G}$; note that $\partial \mathcal{R}_{G}$ is connected by Proposition A.1. By increasing $R_{0}^{\prime}$, if needed, we may assume that $\left\{\left|x_{3}\right| \leq 2\right\} \cap \partial \mathcal{R}_{G} \subset \widetilde{\mathcal{R}}_{S}$. Notice this implies that $\Gamma \cap\left\{\left|x_{3}\right| \leq 2\right\} \subset \widetilde{\mathcal{R}}_{S}=\Sigma^{1} \cup \Sigma^{2}$ and so $\nabla x_{3} \neq 0$ in this set. Moreover, the strict spiraling on $\Sigma^{1} \cup \Sigma^{2}$ guarantees that $\Gamma \cap\left\{x_{3}=c\right\}$, for $|c| \leq 2$, consists of exactly two unbounded, smooth curves with boundary on $\partial \Gamma$.

For $\left\{\left|x_{3}\right| \geq 2\right\}$ we now show that every level set $\left\{x_{3}=c\right\} \cap \Sigma$ consists of one smooth properly embedded curve. We use that $x_{3}$ is harmonic on $\Sigma$, the strict spiraling in $\widetilde{\mathcal{R}}_{S}$ and the proof of Rado's theorem. The key fact is that a non-constant harmonic function $h$ on a closed disk has an interior critical point, $p$, if and only if the connected component of the level set $\{h=h(p)\}$ containing $p$ meets the boundary of the disk in at least 4 points. For $\left|x_{3}\right|>1$, as the genus lies in $B_{1}$, the intersection of $\Sigma$ with wide, short cylinders with axis the $x_{3}$-axis are disks by the maximum principle and Proposition A.1. Moreover, every level set of $x_{3}$ can only have two ends by the strict spiraling. The proof of Rado's theorem then immediately gives the non-vanishing of the gradient for $\left|x_{3}\right|>1$ and so $\nabla_{\Sigma} x_{3} \neq 0$ in $\left|x_{3}\right|>1$. In particular, $\left\{x_{3}=c\right\} \cap \Sigma$ is a smooth curve for $|c|>1$. The final statement of the proposition is then clear. 
In order to show Theorem 1.5, we need only construct $\mathcal{R}_{A}$ and $\mathcal{R}_{S}$ from the $\widetilde{\mathcal{R}}_{A}$ and $\widetilde{\mathcal{R}}_{S}$ of Lemma 3.3 and verify the lower bound on $\left|\nabla_{\Sigma} x_{3}\right|$.

Proof of Theorem 1.5. We first verify that $\left|\nabla_{\Sigma} x_{3}\right|$ is bounded below on $\widetilde{\mathcal{R}}_{A}$. Suppose that $(y, s)$ is a blow-up pair in the sequence constructed in Lemma 3.3 and for convenience rescale so that $s=1$. By our choice of blow-up pairs, we know that every component of $B_{2 \beta R_{1}}(y) \cap \Sigma$ is a disk (where $R_{1}, \beta$ are from Lemma 3.3). Thus, the component of $B_{R_{1}}(y) \cap \Sigma$ containing $y$ is contained in $\mathcal{B}_{\beta R_{1}}(y) \subset B_{2 \beta R_{1}}(y) \cap \Sigma$.

Proposition B.1 implies that curvature is bounded in $B_{2 \beta R_{1}}(y) \cap \Sigma$ by some $K=K\left(R_{1}\right)$. The function $v=-2 \log \left|\nabla_{\Sigma} x_{3}\right| \geq 0$ is smooth by Proposition 1.7 and because, by construction, $B_{2 \beta R_{1}}(y) \cap\left\{\left|x_{3}\right| \leq 1\right\}=\emptyset$. Standard computations give $\Delta_{\Sigma} v=|A|^{2}$. Then, since $\left|\nabla_{\Sigma} x_{3}\right|=1$ somewhere in the component of $B_{R_{1}}(y) \cap \Sigma$ containing $y$, we can apply a Harnack inequality (see Theorems 9.20 and 9.22 in [11]) to obtain an upper bound for $v$ on $\mathscr{B}_{\beta R_{1}}(y)$ that depends only on $K$. Consequently, there is a lower bound $\epsilon_{1}$ on $\left|\nabla_{\Sigma} x_{3}\right|$ in the component of $\Sigma \cap B_{R_{1}}(y)$ containing $y$. Since this bound is scaling invariant, the same bound holds around any blow-up pair from Lemma 3.3.

Recall, $\mathcal{R}_{G}$ is given by $\Sigma \backslash \Gamma$ where $\Gamma$ is from Proposition 1.7. Suppose $\Omega$ is a component of $\Sigma \backslash\left(\mathcal{R}_{G} \cup \widetilde{\mathcal{R}}_{A}\right)$. By the construction of Lemma 3.3, $\Omega$ is either bounded or a subset of $\widetilde{\mathcal{R}}_{S}$. We need consider only bounded $\Omega$. Notice $\partial \Omega \subset \partial\left(\mathcal{R}_{G} \cup \widetilde{\mathcal{R}}_{A}\right) \subset$ $\partial \mathcal{R}_{G} \cup \partial \widetilde{\mathcal{R}}_{A}$. As $\partial \mathcal{R}_{G}$ is compact, and, by construction, $\nabla_{\Sigma} x_{3} \neq 0$ on it, there exists some $\epsilon_{2}>0$ such that $\left|\nabla_{\Sigma} x_{3}\right| \geq \epsilon_{2}>0$ on $\partial \mathcal{R}_{G}$. Let $\epsilon_{0}=\min \left\{\epsilon_{1}, \epsilon_{2}\right\}$. Since $v$ is subharmonic, $\left|\nabla_{\Sigma} x_{3}\right| \geq \epsilon_{0}$ on $\Omega$. Thus, define $\mathcal{R}_{A}$ to be the union of all these $\Omega$ with $\widetilde{\mathcal{R}}_{A} \backslash \mathcal{R}_{G}$. Set $\mathcal{R}_{S}=\Sigma \backslash\left(\mathcal{R}_{A} \cup \mathcal{R}_{G}\right) \subset \widetilde{R}_{S}$.

\section{Conformal structure of the end}

In Section 4.3 we prove Theorem 1.1 and Corollary 1.2 by analysis similar to that in Section 5 of [1]. We first show that $\Gamma=\Sigma \backslash \mathcal{R}_{G}$ is conformally a punctured disk and, indeed, the map $z=x_{3}+i x_{3}^{*}: \Gamma \rightarrow \mathbb{C}$ is a proper, holomorphic coordinate. We then study the level sets. Recall we let $x_{3}^{*}$ denote the harmonic conjugate of $x_{3}$. In order to show that $z$ is a proper, holomorphic coordinate, one must check three things: that $z$ is well defined, that it is injective and that it is proper - i.e., if $p \rightarrow \infty$ in $\Gamma$ then $z(p) \rightarrow \infty$. The first two statements are straightforward, whereas the latter is far more subtle.

Proposition 4.1. $z: \Gamma \rightarrow \mathbb{C}$ is a holomorphic coordinate.

Proof. We first check $x_{3}^{*}$ is well defined on $\Gamma$. As $\Sigma$ is minimal, ${ }^{*} d x_{3}$, the conjugate differential to $d x_{3}$, exists on $\Sigma$ and is closed and harmonic. We wish to show it is exact on $\Gamma$. To do so, it suffices to show that for every embedded closed curve $v$ in 
$\Gamma$, we have $\int_{v}{ }^{*} d x_{3}=0$. By Proposition A.1, $\Sigma \backslash v$ has two components, only one of which is bounded. The bounded component, together with $v$, is a manifold with (connected) boundary, and on this manifold ${ }^{*} d x_{3}$ is a closed form. Hence, the result follows immediately from Stokes' theorem.

We next check that $z$ is injective on $\Gamma$. First notice that, by Proposition 1.7, for any regular value $c$ of $x_{3},\left\{x_{3}=c\right\}$ has exactly one unbounded curve and $x_{3}^{*}$ is strictly monotone along this curve. Now suppose $p, q \in \Gamma, p \neq q$ and $x_{3}(p)=x_{3}(q)$ is a critical value of $x_{3}$. Note that $p$ and $q$ are regular points of $x_{3}-$ as they lie on $\Gamma-$ and so in a neighborhood of each point $z$ is injective. Clearly, there are points $p^{\prime}, q^{\prime} \in \Gamma$ arbitrarily near $p, q$ so that $x_{3}\left(p^{\prime}\right)=x_{3}\left(q^{\prime}\right)=c^{\prime}$ is a regular value of $x_{3}$. Proposition 1.7 implies the unbounded component, $\gamma$, of $\left\{x_{3}=c^{\prime}\right\} \cap \Sigma$ contains $p^{\prime}$ and $q^{\prime}$. The fact that $z$ is injective near $p$ and the monotonicity of $x_{3}^{*}$ on $\gamma$ together give positive lower bound on $\left|x_{3}^{*}\left(p^{\prime}\right)-x_{3}^{*}\left(q^{\prime}\right)\right|$ independent of $p^{\prime}, q^{\prime}$. By continuity, this implies a positive lower bound on $\left|x_{3}^{*}(p)-x_{3}^{*}(q)\right|$ and so $z(p) \neq z(q)$.

4.1. The winding number of the Gauss map. In order to show that $z$ is proper we use the Gauss map of $\Sigma$, or, more accurately, we use $g$, its stereographic projection. In particular, the logarithm of $g$, in $\Gamma$, allows one to prove that $z$ is proper by complex analytic methods. We will make this argument in Section 4.2. However, before we do so we must check such a logarithm is well-defined. Notice as $\Gamma$ is an annulus it is not a priori clear that there exists $f: \Gamma \rightarrow \mathbb{C}$ such that $g=e^{f}$ on $\Gamma$. For such an $f$ to exist we must show that the (topological) winding number of $g$ as a map from the annulus $\Gamma$ to the annulus $\mathbb{S}^{2} \backslash\{ \pm(0,0,1)\}$ is zero. Because $g$ is meromorphic in $\Sigma$ and has no poles or zeros in $\Gamma$, this is equivalent to proving that $g$ has an equal number of poles and zeros.

Proposition 4.2. Counting multiplicity, $g$ has an equal number of poles and zeros.

Proof. The zeros and poles of $g$ occur only at the critical points of $x_{3}$. In particular, by Proposition 1.7, there exist $h$ and $R$ so that all the zeros and poles lie in the cylinder

$$
C_{h, R}=\left\{\left|x_{3}\right| \leq h, x_{1}^{2}+x_{2}^{2} \leq R^{2}\right\} \cap \Sigma .
$$

Moreover, for $R$ and $h$ large, $\gamma=\partial C_{h, R}$ is the union of four smooth curves, two at the top and bottom, $\gamma_{t}$ and $\gamma_{b}$, and two disjoint helix like curves $\gamma_{1}, \gamma_{2} \subset \mathcal{R}_{S}$. Hence, for $c \in(-h, h),\left\{x_{3}=c\right\}$ meets $\partial C_{h, R}$ in exactly two points. Additionally, as $\gamma_{1}$ and $\gamma_{2}$ are compact, there is a constant $\alpha>0$ such that $\left|\frac{d}{d t} x_{3}\left(\gamma_{i}(t)\right)\right|>\alpha$, $i=1,2$.

Let us first suppose that $g$ has only simple zeros and poles and these occur at distinct values of $x_{3}$; thus, the Weierstrass representation implies that the critical points of $x_{3}$ are non-degenerate. We now investigate the level sets $\left\{x_{3}=c\right\}$. By the strict spiraling of $\gamma_{i}(i=1,2)$, at the regular values these level sets consist of an 
interval with end points in $\gamma_{i}(i=1,2)$ and the union of a finite number of closed curves. Moreover, by the minimality of $C_{h, R}$, the non-smooth components of the level sets at critical values will consist of either two closed curves meeting in a single point or the interval and a closed curve meeting in a single point. As a consequence of this $\left\{\left|x_{3}\right| \leq h, x_{1}^{2}+x_{2}^{2} \leq R^{2}\right\} \backslash C_{h, R}$ has exactly two connected components $\Omega_{1}$ and $\Omega_{2}$. Orient $C_{h, R}$ by demanding that the normal point into $\Omega_{1}$. Notice it is well defined to say if a closed curve appearing in $\left\{x_{3}=c\right\} \cap C_{h, R}$ surrounds $\Omega_{1}$ or $\Omega_{2}$.

The restrictions imposed on $g$ and minimality of $C_{h, R}$ imply that at any critical level, as one goes downward, either a single closed curve is "created" or is "destroyed". (See Figure 4.) Moreover, when such a curve is created it makes sense to say whether it surrounds $\Omega_{1}$ or $\Omega_{2}$ and this is preserved as one goes downward. Now suppose a closed curve is created and that it surrounds $\Omega_{1}$; then it is not hard to see that at the critical point the normal must point upwards. Similarly, if a closed curve surrounding $\Omega_{1}$ is destroyed then the normal at the critical point is downward pointing. For closed curves surrounding $\Omega_{2}$ the opposite is true; e.g. when a closed curve is created, then at the critical point the normal points downward. Thus, since the level sets at $h$ and $-h$ are intervals, one sees that the normal points up as much as it points down. That is, $g$ has as many zeros as poles.

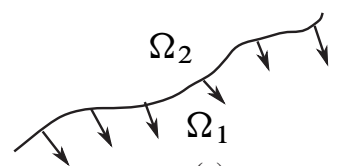

(a)

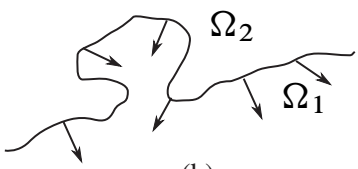

(b)

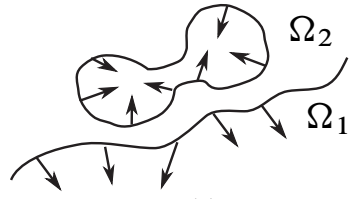

(c)

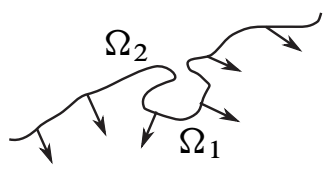

(d)

Figure 4. Level curve examples in Proposition 4.2. (a) Initial orientation chosen at height $x_{3}=h$. (b) A curve pinching off from $\Omega_{1}$. (c) Two curves pinching from one. (d) A curve pinching off from $\Omega_{2}$.

We now drop the restrictions on the poles and zeros of $g$. Beyond these assumptions the argument above used only that $C_{h, R}$ was minimal and that the boundary curves $\gamma_{i}(i=1,2)$ meet the level curves of $x_{3}$ in precisely one point. It is not hard to check that these last two conditions are preserved by small rotations around lines in the $x_{1}-x_{2}$ plane. We claim that such rotations also ensure that the Gauss map of the new surface must have simple poles or zeros and these are on distinct level sets. 
To that end we let $C_{h, R}^{\epsilon}$ be the rotation of $C_{h, R}$ by $\epsilon$ degrees around a fixed line $\ell$ in the $x_{1}-x_{2}$ plane and through the origin.

The strict spiraling of $\gamma_{1}, \gamma_{2}$ implies there exists an $\epsilon_{0}>0$, depending on $\alpha$ and $R$ and a constant $K>0$, depending on $R$, so that for all $0<\epsilon<\epsilon_{0}$, if $c \in(-h+K \epsilon, h-K \epsilon)$ then $\left\{x_{3}=c\right\} \cap C_{h, R}^{\epsilon}$ meets $\partial C_{h, R}^{\epsilon}$ in two points. Moreover, by a suitable choice of $\ell$ the critical points will be on distinct level sets. Denote by $g_{\epsilon}$ the stereographic projection of the Gauss map of $C_{h, R}^{\epsilon}$. We now use the fact that $g$ is meromorphic on $\Sigma$ (and thus the zeros and poles of $g$ are isolated) and that $g_{\epsilon}$ is obtained from $g$ by a Möbius transform. Indeed, these two facts imply that (after shrinking $\left.\epsilon_{0}\right)$ for $\epsilon \in\left(0, \epsilon_{0}\right), g_{\epsilon}$ has only simple zeros and poles on $C_{h, R}^{\epsilon}$ and by our choice of $\ell$ these are on distinct levels of $x_{3}$. To see this we note that there are $\alpha(\epsilon), \beta(\epsilon) \in \mathbb{C}$ (and also depending on $\ell$ ) satisfying $|\alpha(\epsilon)|^{2}+|\beta(\epsilon)|^{2}=1$ so that

$$
g_{\epsilon}=\frac{\alpha(\epsilon) g-\bar{\beta}(\epsilon)}{\beta(\epsilon) g+\bar{\alpha}(\epsilon)},
$$

where we have also $|\alpha(\epsilon)| \neq 0,1$ and $\alpha(\epsilon) \rightarrow 1$ as $\epsilon \rightarrow 0$. Thus, for $\epsilon$ sufficiently small all zeros of $g_{\epsilon}$ are distinct from, but near, zeros of $g$. This implies that, at the zeros of $g_{\epsilon}, d g_{\epsilon}$ does not vanish.

By further shrinking $\epsilon_{0}$ one can ensure that all of the critical values occur in the range $(-h+K \epsilon, h-K \epsilon)$. Thus, the level sets in $C_{h, R}^{\epsilon}$ of $x_{3}$ for $c \in(-h+K \epsilon, h-K \epsilon)$ consist of an interval with endpoints in $\partial C_{h, R}^{\epsilon}$, one in each $\gamma_{i}$ for $i=1,2$, and the union of a finite number of closed curves. Our original argument then immediately implies that $g_{\epsilon}$ has as many zeros as poles. Notice this is equivalent to the vanishing of the winding number of the map $g_{\epsilon}$ restricted to $\partial C_{h, R}^{\epsilon}$ (which is topologically $\mathbb{S}^{1}$ ) as a map into $\mathbb{C} \backslash\{0\}$. For $\epsilon$ sufficiently small, $g_{\epsilon}$ never has a zero or pole on $\partial C_{h, R}^{\epsilon}$ and as long as this is true, the winding number is independent of $\epsilon$. Thus, $g$ has, counting multiplicity, the same number of poles and zeros.

Corollary 4.3. A holomorphic function $f: \Gamma \rightarrow \mathbb{C}$ exists such that $e^{f}=g$ on $\Gamma$.

4.2. The conformal structure of the end. The strict spiraling in $\mathcal{R}_{S}$ is used in [1] to show that the logarithm of $g$, i.e. $f=f_{1}+i f_{2}$, is, away from a neighborhood of $\mathcal{R}_{A}$, a proper conformal diffeomorphism onto the union of two disjoint closed halfspaces. Since every level set of $x_{3}$ has an end in each of these sets, the properness of $z$ was then a consequence of Schwarz reflection and the Liouville theorem. The proof only used properties of the end of the surface and so holds also in $\mathscr{E}(1,+)$ :

Proposition 4.4. There exists a $\gamma_{0}>0$ such that $f$ is a proper conformal diffeomorphism from $\Omega_{ \pm}$onto $\left\{z: \pm \operatorname{Re} z \geq 2 \gamma_{0}\right\} \subset \mathbb{C}$, where

$$
\Omega_{ \pm}=\left\{x \in \Gamma: \pm f_{1}(x) \geq 2 \gamma_{0}\right\} \subset \Gamma .
$$


Proposition 5.1 in [1] asserts and proves the identical statement for minimal disks. The proof relies on showing there exists $\gamma_{0}$ such that for every regular value $\gamma \geq 2 \gamma_{0}$, $f_{1}^{-1}(\gamma)$ consists of exactly one curve on $\Sigma$, which lies on every sheet of one of the components of $\mathcal{R}_{S}$ (note in [1] $\log g$ is denoted by $h$ ). We rely on the fact that $\left|\nabla_{\Sigma} x_{3}\right|$ is a function of $\left|f_{1}\right|$. Recall, $\nabla_{\Sigma} x_{3}$ is the projection of $\mathbf{e}_{3}=\nabla_{\mathbb{R}^{3}} x_{3}$ onto $T \Sigma$, and so $\left|\nabla_{\Sigma} x_{3}\right|$ can be expressed terms of the $x_{3}$-coordinate of the unit normal to $T \Sigma$. Thus, by computing the inverse stereographic projection, one obtains

$$
\left|\nabla_{\Sigma} x_{3}\right|=2 \frac{|g|}{1+|g|^{2}} \leq 2 e^{-\left|f_{1}\right|} .
$$

By Theorem 1.5, as $\left|\nabla_{\Sigma} x_{3}\right| \geq \epsilon_{0}>0$ on $\mathcal{R}_{A} \cup \partial \mathcal{R}_{G}$, there exists $\gamma_{0}>0$ such that $\left|f_{1}(z)\right| \leq \gamma_{0}$ on $\mathcal{R}_{A} \cup \partial \mathcal{R}_{G}$. The proof in [1] only requires that $f_{1}^{-1}(\gamma)$ lies in $\mathcal{R}_{S}$; thus, since $f_{1}^{-1}\left(2 \gamma_{0}\right) \cap \partial \Gamma=\varnothing$, using $f_{1}^{-1}(\gamma) \cap \Gamma$, the proof carries over without change. The interested reader should consult Proposition 5.1 in [1] for the details.

4.3. The proofs of Theorem 1.1 and Corollary 1.2. In Proposition 5.2 of [1], we show that for $\Sigma \in \mathcal{E}(1,0), f \circ z^{-1}: \mathbb{C} \rightarrow \mathbb{C}$ is linear. The result follows from standard complex analysis, exploiting both Schwarz reflection and Liouville's theorem. For $\Sigma \in \mathcal{E}(1,+)$, there are a few necessary, but simple, modifications.

Proof of Theorem 1.1. We first show that $x_{3}^{*} \rightarrow \pm \infty$ along each level set of $x_{3}$; that is $z: \Gamma \rightarrow \mathbb{C}$ is a proper holomorphic coordinate. This follows easily once we establish that each level set of $x_{3}$ has one end in $\Omega_{+}$and the other in $\Omega_{-}$, where these sets are as defined in (4.3). This follows from the radial gradient decay on level sets of $x_{3}$ forced by the one-sided curvature estimate. Indeed, Corollary C.3 and the structural decomposition of $\Sigma$ imply that for any $\epsilon>0$ small, there is a point $y_{\epsilon} \in \Sigma$ and a $\delta_{\epsilon}>0$ so that within suitable subsets of $\mathbf{C}_{\delta_{\epsilon}}\left(y_{\epsilon}\right), \Sigma$ must be a graph with gradient bounded by $\epsilon$. Recall (2.5) says that, for any $\delta_{\epsilon}$-sheet, there is sub-linear gradient decay on the sheet and so it must eventually lie within $\mathbf{C}_{\delta_{\epsilon}}\left(y_{\epsilon}\right)$. Thus, by Corollary C.3 for some large $R_{\epsilon}>0$ every point of $\Sigma \cap \mathbf{C}_{\delta_{\epsilon}}\left(y_{\epsilon}\right) \backslash B_{R_{\epsilon}}\left(y_{\epsilon}\right)$ lies on some multi-valued graph that has gradient bounded by $\epsilon$. Notice any level set of $x_{3}$ has its ends in this set and so $\left|\nabla_{\Sigma} x_{3}\right| \leq C \epsilon$ in a neighborhood of the ends.

Thus, $x_{3}\left(\partial \Omega_{+}\right)=(-\infty, \infty)$ and so $z\left(\partial \Omega_{+}\right)$splits $\mathbb{C}$ into two components with only one, $V$, meeting $z\left(\Omega_{+}\right)=U$. If $U$ is a proper subset of $V$ then, by conformally straightening the boundary of $V$ and precomposing with $f^{-1} \mid \Omega_{+}$, we can apply Schwarz reflection to get a map from $\mathbb{C}$ into a proper subset of $\mathbb{C}$. The Liouville theorem then implies that $z$ is constant. This gives a contradiction and so $U=V$, i.e., $x_{3}^{*} \rightarrow \pm \infty$ along each level set of $x_{3}$. Thus, $z(\Gamma)$ contains $\mathbb{C}$ with a closed disk removed; in particular, $\Gamma$ is conformally a punctured disk. Since $f_{1}^{-1}\left(\gamma_{0}\right) \cap$ $\Gamma$ is a single smooth curve, $f$ has a simple pole at the puncture. Similarly, by Proposition 1.7, $z$ has a simple pole at the puncture. In $\Gamma$, the height differential $d h=d z$ and $\frac{d g}{g}=d f$, proving the theorem. 
Embeddedness and the Weierstrass representation (1.1) then give Corollary 1.2:

Proof of Corollary 1.2. Theorem 1.1 gives that, in $\Gamma, f(p)=\alpha z(p)+\beta+F(p)$ where $\alpha, \beta \in \mathbb{C}$ and $F: \Gamma \rightarrow \mathbb{C}$ is holomorphic and has holomorphic extension to the puncture (and has a zero there). By translating $\Sigma$ parallel to the $x_{3}$-axis and re-basing $x_{3}^{*}$ we may assume $\beta=0$. By Proposition $1.7,\left\{x_{3}=0\right\} \cap \Gamma \subset \mathcal{R}_{S}$ can be written as the union of two smooth proper curves, $\sigma_{ \pm}$, each with one end in $\partial \Gamma$, and parametrized such that $x_{3}^{*}\left(\sigma_{ \pm}(t)\right)=t$ for $\pm t>T$.

Note that, since $\sigma_{ \pm}^{\prime}(t)$ is perpendicular to both $\mathbf{e}_{3}$ and to the normal $\mathbf{n}$ to $\Sigma$ at $\sigma_{ \pm}(t)$, the projection of $\mathbf{n}$ onto the $\left\{x_{3}=0\right\}$ plane is also perpendicular to $\sigma_{ \pm}^{\prime}(t)$. This projection is, up to the correct identification of $\left\{x_{3}=0\right\}$ with $\mathbb{C}$, parallel to $g\left(\sigma_{ \pm}(t)\right)$. Since $\arg \left(g\left(\sigma_{ \pm}(t)\right)\right)=(\operatorname{Re} \alpha) t+\operatorname{Im} F\left(\sigma^{ \pm}(t)\right)$, we see that $\arg \left(\sigma_{ \pm}^{\prime}(t)\right)=$ $\pm \pi / 2+(\operatorname{Re} \alpha) t+o(1 / t)$. Consider, for a moment, the curve $\sigma_{+}(t)$. If $\operatorname{Re} \alpha \neq 0$, $\arg \left(\sigma_{+}^{\prime}(t)\right) \rightarrow \infty$ as $t \rightarrow \infty$. Thus, $\sigma_{+}$hits the $x_{1}$-axis infinitely many times. As $\Sigma$ is properly embedded, this set of intersections tends to $\infty$. Note that the same can be said for $\sigma_{-}$, but the choice of parametrization means it spirals in the opposite direction. Thus, the two curves must intersect which contradicts embeddedness. Therefore, $\operatorname{Re} \alpha=0$.

\section{Appendix}

\section{A. Topological structure of $\Sigma$}

An elementary but crucial consequence of the maximum principle is that each component of the intersection of a minimal disk with a closed ball is a disk. Similarly, each component of the intersection of a genus $k$ surface with a ball has genus at most $k$ (see Appendix C of [7] and Section I of [6]). We note that for $\Sigma$ with one end and finite genus we obtain a bit more:

Proposition A.1. Suppose $\Sigma \in \mathcal{E}(1)$ and $\bar{\Sigma} \subset \Sigma \cap B_{1}$ is smooth and connected, with the same genus as $\Sigma$. Then, $\Sigma \backslash \bar{\Sigma}$ is an annulus. Moreover, for any convex set $C$ with non-empty interior, if $C \cap B_{1}=\emptyset$, then each component of $C \cap \Sigma$ is a disk. Alternatively, if $B_{1} \subset C$ then all components of $C \cap \Sigma$ not containing $\bar{\Sigma}$ are disks.

Proof. That $\Sigma^{\prime}=\Sigma \backslash \bar{\Sigma}$ is an annulus is a purely topological consequence of $\Sigma$ having one end. Indeed, as the Euler characteristic satisfies $\chi(\Sigma)=\chi\left(\Sigma^{\prime}\right)+\chi(\bar{\Sigma})$ and $\Sigma$ has one end, one computes that $2 g\left(\Sigma^{\prime}\right)+e\left(\Sigma^{\prime}\right)+e(\bar{\Sigma})=3$, where $g(X)$ and $e(X)$ respectively represent the genus and number of punctures of $X$. On the other hand, as $\Sigma$ has one end, $e\left(\Sigma^{\prime}\right)=1+e(\bar{\Sigma})$ proving the claim.

If $C$ and $B_{1}$ are disjoint then, as they are convex, there exists a plane $P$ so that $P$ meets $\Sigma$ transversely and so that $P$ separates $B_{1}$ and $C$. Since $\Sigma \backslash \bar{\Sigma}$ is an annulus and $P \cap \bar{\Sigma}=\emptyset$, the convex hull property implies that $P \cap \Sigma$ consists only of unbounded 
smooth proper curves. Thus, exactly one of the components of $\Sigma \backslash P$ is not a disk. As $C$ is disjoint from this component we have the desired result.

If $C$ is convex and contains $B_{1}$, denote by $\Gamma$ the component of $C \cap \Sigma$ containing $\bar{\Sigma}$. Suppose $\Gamma_{1}$ is a different component of $\Sigma \cap C$. Let $\gamma$ be a component of $\partial \Gamma_{1}$. As $\Sigma \backslash \bar{\Sigma}$ is an annulus, we have that $\gamma$ is separating, in particular, one component, $\Gamma_{2}$ of $\Sigma \backslash \gamma$ is pre-compact. Clearly, $\Gamma_{2}$ either contains $\bar{\Sigma}$ or is a disk. By the convex hull property, one has that $\Gamma_{2} \subset C$ and so $\Gamma_{2}$ is a component of $\Sigma \cap C$. Thus, by the strong maximum principle $\Gamma_{1}=\Gamma_{2}$ and so $\Gamma_{1}$ is a disk.

\section{B. Geometry near a blow-up pair}

The existence of a blow-up pair $(y, s)$ in a minimal surface $\Sigma$, by definition, implies uniform bounds on the geometry in the ball $B_{s}(y)$. Colding and Minicozzi's work shows further that there are uniform bounds on the geometry in any ball on the scale of $s$. This is most easily proved using their lamination results. Indeed, we have the following uniform bound on the curvature, which is an extension of Lemma 2.26 of [8] to surfaces of finite genus:

Proposition B.1. Given $K_{1}, g$ we get a constant $K_{2}$ such that if

(1) $\Sigma \subset \mathbb{R}^{3}$ is an embedded minimal surface with genus $(\Sigma)=g$,

(2) $\Sigma \subset B_{K_{2} s}(y)$ and $\partial \Sigma \subset \partial B_{K_{2} s}(y)$,

(3) $(y, s)$ is a blow-up pair,

then we get the curvature bound

$$
\sup _{B_{K_{1} s}(y) \cap \Sigma}|A|^{2} \leq K_{2} s^{-2} .
$$

The proof is nearly identical to that of Lemma 2.26 of [8]. That proof is by contradiction, using Colding and Minicozzi's compactness result for minimal disks, i.e. Theorem 0.1 of [7]. One proves Proposition B.1 by the same argument, but uses instead a more general compactness result, i.e. Theorem 0.6 of [9].

\section{One-sided curvature in $\Sigma$}

In several places we make use of the one-sided curvature estimate of [7]. Recall that this result gives a curvature estimate for a minimal disk that is close to and on one side of a plane. As a sequence of rescaled catenoids shows, it is crucial that the surface be a disk, something that makes application to surfaces in $\mathscr{E}(1,+)$ somewhat subtle. Nevertheless, Proposition A.1 allows the use of the one-sided curvature estimate far from the genus. Recall the statement of the estimate: 
Theorem C.1 (Theorem 0.2 of [7]). There exists $\epsilon>0$ so that if $\Sigma \subset B_{2 r_{0}} \cap$ $\left\{x_{3}>0\right\} \subset \mathbb{R}^{3}$ is an embedded minimal disk with $\partial \Sigma \subset \partial B_{2 r_{0}}$, then for all components $\Sigma^{\prime}$ of $\Sigma \cap B_{r_{0}}$ which intersect $B_{\epsilon r_{0}}$ we have

$$
\sup _{\Sigma^{\prime}}\left|A_{\Sigma}\right|^{2} \leq r_{0}^{-2}
$$

A particularly important consequence of Theorem C.1 is Corollary I.1.9 of [7], which roughly states that if an embedded minimal disk has a two-valued graph in the complement of a cone (and away from a ball), then all components of $\Sigma$ in the complement of a larger cone (and larger ball) are multi-valued graphs. Essentially, the two-valued graph takes the place of the plane in Theorem C.1. This fact was used extensively in [1]. Thus, we give two variants of it that hold for elements of $\mathscr{E}(1,+)$ and indicate how they follow from [7].

Corollary C.2. There exists a $c>1$ such that for a given $\epsilon>0$ there exists $\delta_{0}>0$ such that if $\mathbf{C}_{c \delta_{0}}(y) \backslash B_{s}$ does not meet $\bar{\Sigma}$ and contains a weak 2-valued $\delta_{0}$-sheet centered at $y$ and on scale s, then each component of $\Sigma \cap\left(\mathbf{C}_{\delta_{0}}(y) \backslash B_{2 s}(y)\right)$ is a multi-valued graphs with gradient bounded by $\epsilon$.

Proof. The result follows immediately from the proof of Corollary I.1.9 of [7] (as long as one notes that the proof of Corollary I.1.9 depends only on each component of $\Sigma \cap\left(\mathbf{C}_{c \delta_{0}}(y) \backslash B_{s(y)}\right)$ meeting any convex set in a disk (and so Theorem C.1 applies) for $c$ a universal constant. Proposition A.1 and the hypothesis ensure this.

We will also use the following specialization of the above:

Corollary C.3. Given $\epsilon, \delta>0$ there exist $\delta_{0}>0$ and $R>1$ such that, if there exists a weak 2-valued $\delta_{0}$-sheet centered at $y$ on scale s where $y \notin \mathbf{C}_{\delta} \cup B_{R}$, then all the components of $\Sigma \cap\left(\mathbf{C}_{\delta_{0}}(y) \backslash B_{2 s}(y)\right)$ are multi-valued graphs with gradient $\leq \epsilon$.

Proof. The result follows immediately from Corollary C.2 as long as we can ensure that $\bar{\Sigma} \subset B_{1}(0)$, is disjoint from $\mathbf{C}_{c \delta_{0}}(y) \backslash B_{s}(y)$. Suppose $x \in \mathbf{C}_{c \delta_{0}}(y)$ and think of $x$ and $y$ as vectors. By choosing $\delta_{0}$ sufficiently small, depending on $\delta$, we have that $|\langle x-y, y\rangle|<(1-\gamma)|y||x-y|$ (that is the angle between $x-y$ and $y$ is bounded away from $0^{\circ}$ ); note $1>\gamma>0$ depends only on $\delta$. But then $|x|^{2}=|x-y+y|^{2} \geq$ $|x-y|^{2}+2\langle x-y, y\rangle+|y|^{2} \geq \gamma|y|^{2}$. Hence, picking $R^{2}>\frac{1}{\gamma}$ suffices.

\section{Colding-Minicozzi lamination theory}

We note that Theorem 1.5 is a sharpening, for $\Sigma \in \mathcal{E}(1)$, of a much more general description of the shapes of minimal surfaces given by Colding and Minicozzi in [9]. 
More precisely, in that paper they show, for a large class of embedded minimal surfaces in $\mathbb{R}^{3}$, how the geometric structure of a surface is determined by its topological properties. In particular, as $\Sigma$ has finite topology and one end, their work shows that it roughly looks like a helicoid. That is, away from a compact set containing the genus, $\Sigma$ is made up of two infinite-valued graphs that spiral together and are glued along an axis. Using this description, they show compactness results that generalize their lamination theory of [7]. As in the case for disks, the derivation of this global description of finite genus surfaces uses local versions of propositions as in Section 2.2. However, Colding and Minicozzi do not explicitly state these results and so, for the sake of completeness, we will state a modified form of a compactness result from [9] and use it to give simple proofs of Propositions 2.5 and 2.6.

While the lamination theory of [9] will be the launching point for proving the two propositions, we need only outline one small portion of the theory to get our result. In particular, we need only consider the structure of the limit lamination of homothetic dilations for $\Sigma \in \mathscr{E}(1)$. In this case, the lamination has the same structure as for a sequence of embedded minimal disks, which is modeled on rescalings of the helicoid.

Theorem D.1. Let $\Sigma \in \mathcal{E}(1)$ be non-flat, and let $\lambda_{i} \rightarrow 0$. Set $\Sigma_{i}=\lambda_{i} \Sigma$. There exists a subsequence $\Sigma_{j}$, a foliation $\mathscr{L}=\left\{x_{3}=t\right\}_{t \in \mathbb{R}}$ of $\mathbb{R}^{3}$ by parallel planes, and a closed nonempty set $S$ in the union of the leaves of $\mathscr{L}$ such that after a rotation of $\mathbb{R}^{3}$ :

(1) For each $1>\alpha>0, \Sigma_{j} \backslash S$ converges in the $C^{\alpha}$-topology to the foliation $\mathscr{L} \backslash S$.

(2) $\sup _{B_{r}(x) \cap \Sigma_{j}}|A|^{2} \rightarrow \infty$ as $j \rightarrow \infty$ for all $r>0$ and $x \in \mathcal{S}$. (The curvatures blow up along $S$.)

(3) Away from $\mathcal{S}$, each $\Sigma_{j}$ consists of exactly two multi-valued graphs spiraling together.

(4) $S$ is a single line orthogonal to the leaves of the foliation.

Remark D.2. For the theorem in its entirety, see Theorem 0.9 of [9].

We now use the nature of this convergence to deduce gradient bounds outside a cone. This, together with further application of the compactness theorem, gives Propositions 2.5 and 2.6.

Lemma D.3. For any $\epsilon>0, \delta>0$ there exists an $R>1$ such that every component of $\left(\mathbf{C}_{\delta} \backslash B_{R}\right) \cap \Sigma$ is a graph over $\left\{x_{3}=0\right\}$ with gradient less than $\epsilon$.

Proof. We proceed by contradiction. Suppose there exists a sequence $\left\{R_{i}\right\}$ with $R_{i} \rightarrow \infty$ and points $p_{i} \in\left(\mathbf{C}_{\delta} \backslash B_{R_{i}}\right) \cap \Sigma$ such that the component of $B_{\gamma\left|p_{i}\right|}\left(p_{i}\right) \cap \Sigma$ containing $p_{i}, \Omega_{i}$, is not a graph over $\left\{x_{3}=0\right\}$ with gradient less than $\epsilon$. Here $\gamma$ 
depends on $\delta$ and will be specified later. Now, consider the sequence of rescalings $\frac{1}{\left|p_{i}\right|} \Sigma$, which by possibly passing to a subsequence converges to $\mathscr{L}$ (away from $\mathcal{S}$ ). Passing to another subsequence, $\frac{1}{\left|p_{i}\right|} p_{i}$ converges to a point $p_{\infty} \in \mathbf{C}_{\delta} \cap B_{1}$. Let $\widetilde{\Omega}_{i}=\frac{1}{\left|p_{i}\right|} \Omega_{i}$. Proposition III.0.2 of [9] guarantees that if $B_{\gamma}\left(p_{\infty}\right) \cap S=\emptyset$ then the $\widetilde{\Omega}_{i}$ converge to $\widetilde{\Omega}_{\infty} \subset\left\{x_{3}=x_{3}\left(p_{\infty}\right)\right\}$ as graphs. As $\delta$ is the sole singular set, we may choose $\gamma$ small, depending only on $\delta$, to ensure this. Thus, for large $j, \widetilde{\Omega}_{j}$ is a graph over $\left\{x_{3}=0\right\}$ with gradient bounded by $\epsilon$, giving the desired contradiction.

We now show Propositions 2.5 and 2.6:

Proof of Proposition 2.5. Let $\tilde{\delta}=\epsilon$ and $\tilde{\epsilon} \leq \tilde{\delta} /(4 \pi N)$. Choose $R$ from Lemma D.3, using this $\tilde{\delta}, \tilde{\epsilon}$. Thus, every component of $\Sigma \cap \mathbf{C}_{\tilde{\delta}} \backslash B_{R}$ has gradient bounded by $\tilde{\epsilon}$. Since $|w(\rho, \theta)| \leq \int_{0}^{2 \pi}\left|u_{\theta}\right| \leq 2 \pi \tilde{\epsilon} \rho$, we see that there are $N$ sheets in $\mathbf{C}_{\epsilon}$.

Proof of of Proposition 2.6. Note that as long as $|y|$ is sufficiently large, Theorem 0.6 of [5] gives an $\Omega<1 / 2$ (as well as a constant $C_{1}$ ) so that since the component of $B_{\frac{1}{2}|y|}(y) \cap \Sigma$ containing $y$ is a disk, there exists a $N$-valued graph $\Sigma_{0}$ over the annulus, $A=D_{\Omega|y|} \backslash D_{s / 2}(y) \subset P$ with gradient bounded by $\epsilon / 2$. Here $P$ is in principle an arbitrary plane in $\mathbb{R}^{3}$.

We claim that Lemma D.3 implies a subset, $\Sigma_{0}^{\prime}$, of $\Sigma_{0}$ is a $N$-valued graph over the annulus $A^{\prime}=D_{\Omega|y| / 2} \backslash D_{s}(\Pi(y)) \subset\left\{x_{3}=0\right\}$ with gradient bounded by $\epsilon$, which further implies $\Sigma_{0}^{\prime}$ can be extended as desired. To that end we note that for $\delta>1 /(4 \Omega)$, if $y \notin \mathbf{C}_{\delta}$ then $A$ (and thus, by possibly increasing $\left.\delta, \Sigma_{0}\right)$ meets $\mathbf{C}_{\delta}$. Lemma D.3 allows us to choose an $R_{0}>0$ so that every component of $\Sigma \cap\left(\mathbf{C}_{\delta} \backslash B_{R_{0}}\right)$ is a multi-valued graph over $\left\{x_{3}=0\right\}$ with gradient bounded by $\epsilon / 4$. Thus if we take $R>2 R_{0}$ then there is a point of $\Sigma_{0}$ in $\mathbf{C}_{\delta} \backslash B_{R_{0}}$; therefore, for the gradient estimates at the point to be consistent, $P$ must be close enough to $\left\{x_{3}=0\right\}$ so that we may choose $\Sigma_{0}^{\prime} \subset \Sigma_{0}$ such that it is a multi-valued graph over $A^{\prime}$. Furthermore, the part of $\Sigma_{0}^{\prime}$ over the outer boundary of $A^{\prime}$ is necessarily inside of $\mathbf{C}_{\delta} \backslash B_{R_{0}}$ and so Lemma D.3 allows us to extend it as desired.

\section{References}

[1] J. Bernstein and C. Breiner, Helicoid-like minimal disks and uniqueness. J. Reine Angew. Math., to appear. arXiv:0802.1497v3 [math.DG] 353, 355, 357, 359, 360, 363, 364, 366, $367,368,370,373,374,377$

[2] T. H. Colding and W. P. Minicozzi II, Multivalued minimal graphs and properness of disks. Internat. Math. Res. Notices 2002 (2002), no. 21, 1111-1127. Zbl 1008.58012 MR 1904463 360, 367

[3] T. H. Colding and W. P. Minicozzi II, An excursion into geometric analysis. Surv. Differ. Geom. IX (2004), 83-146. Zbl 1076.53001 MR 2195407 357 
[4] T. H. Colding and W. P. Minicozzi II, The space of embedded minimal surfaces of fixed genus in a 3-manifold I; Estimates off the axis for disks. Ann. of Math. (2) 160 (2004), no. 1, 27-68. Zbl 1070.53031 MR 2119717 354, 357, 361

[5] T. H. Colding and W. P. Minicozzi II, The space of embedded minimal surfaces of fixed genus in a 3-manifold II; Multi-valued graphs in disks. Ann. of Math. (2) 160 (2004), no. 1, 69-92. Zbl 1070.53032 MR 2119718 354, 357, 361, 363, 365, 379

[6] T. H. Colding and W. P. Minicozzi II, The space of embedded minimal surfaces of fixed genus in a 3-manifold III; Planar domains. Ann. of Math. (2) 160 (2004), no. 2, 523-572. Zbl 1076.53068 MR 2123932 354, 357, 364, 365, 366, 367, 375

[7] T. H. Colding and W. P. Minicozzi II, The space of embedded minimal surfaces of fixed genus in a 3-manifold IV; Locally simply connected. Ann. of Math. (2) 160 (2004), no. 2, 573-615. Zbl 1076.53069 MR 2123933 355, 357, 362, 363, 364, 365, 375, 376, 377, 378

[8] T. H. Colding and W. P. Minicozzi II, The Calabi-Yau conjectures for embedded surfaces. Ann. of Math. (2) 167 (2008), no. 1, 211-243. Zbl 1142.53012 MR 2373154 353, 355, $362,365,367,368,376$

[9] T. H. Colding and W. P. Minicozzi II, The space of embedded minimal surfaces of fixed genus in a 3-manifold V; Fixed genus. Preprint. arXiv:0509647v1 [math.DG] 355, 357, $361,376,377,378,379$

[10] P. Collin, Topologie et courboure des surfaces minimales proprement plongées de $\mathbb{R}^{3}$. Ann. of Math. (2) 145 (1997), 1-31. Zbl 0886.53008 MR 1432035356

[11] D. Gilbarg and N. S. Trudinger, Elliptic partial differential equations of second order. Classics Math., Springer-Verlag, Berlin 2001. Zbl 1042.35002 MR 1814364370

[12] L. Hauswirth, J. Perez, and P. Romon, Embedded minimal ends of finite type. Trans. Amer. Math. Soc. 353 (2001), no. 4, 1335-1370. Zbl 0986.53005 MR 1806738 355, 356

[13] D. Hoffman and W. H. Meeks III, The strong halfspace theorem for minimal surfaces. Invent. Math. 101 (1990), no. 1, 373-377. Zbl 0722.53054 MR 1062966355

[14] D. Hoffman, H. Karcher, and F. Wei, The genus one helicoid and the minimal surfaces that led its discovery. In Global analysis in modern mathematics, Publish or Perish, Houston, TX, 1993, 119-170. Zbl 1049.53502 MR 1278754 353, 354

[15] D. Hoffman and J. McCuan, Embedded minimal ends asymptotic to the Helicoid. Commun. Anal. Geom. 11 (2003), no. 4, 721-736. Zbl 1079.53016 MR 2015173355

[16] D. Hoffman, M. Weber, and M. Wolf, An embedded genus-one helicoid. Ann. of Math. (2) 169 (2009), no. 2, 347-448. Zbl 05578747 MR 2480608 353, 354

[17] D. Hoffman, M. Weber, and M. Wolf, The genus-one helicoid as a limit of screw-motion invariant helicoids with handles. In Global theory of minimal surfaces, Clay Math. Proc. 2, Amer. Math. Soc., Providence, RI, 2005, 243-258. Zbl 1102.53008 MR 2167262354

[18] D. Hoffman and F. Wei, Deforming the singly periodic genus-one helicoid. Experiment. Math. 11 (2002), no. 2, 207-218. Zbl 1116.53301 MR 1959264354

[19] D. Hoffman and B. White, Genus-one helicoids from a variational point of view. Comment. Math. Helv. 83 (2008), no. 4, 767-813. Zbl 1161.53009 MR 2442963354

[20] D. Hoffman and B. White, The geometry of genus-one helicoids. Comment. Math. Helv. 84 (2009), 547-569. Zbl 1168.53006 MR 2507253354 
[21] A. Huber, On subharmonic functions and differential geometry in the large. Comment. Math. Helv. 32 (1958), no. 1, 13-72. Zbl 0080.15001 MR 0094452356

[22] W. H. Meeks III and H. Rosenberg, The geometry and conformal structure of properly embedded minimal surfaces of finite topology in $\mathbb{R}^{3}$. Invent. Math. 114 (1993), no. 3, 625-639. Zbl 0803.53007 MR 1244914356

[23] W. H. Meeks III and H. Rosenberg, The uniqueness of the helicoid. Ann. of Math. (2) 161 (2005), no. 2, 727-758. Zbl 1102.53005 MR 2153399 353, 355

[24] R. Osserman, A survey of minimal surfaces. Dover Publications, New York 1986. Zbl 0209.52901 MR 0852409 356, 369

[25] T. Rado, On the problem of Plateau. Ergeb. Math. Grenzgeb. 2, Springer-Verlag, Berlin 1933. JFM 59.1341.01369

[26] M. Weber, D. Hoffman, and M. Wolf, An embedded genus-one helicoid. Proc. Nat. Acad. Sci. 102 (2005), no. 46, 16566-16568. 353

Received October 24, 2008

Jacob Bernstein, Department of Mathematics, Stanford University, Stanford, CA 94305, U.S.A.

E-mail: jbern@math.stanford.edu

Christine Breiner, Department of Mathematics, Massachusetts Institute of Technology, Cambridge, MA 02139, U.S.A.

E-mail: breiner@mit.edu 